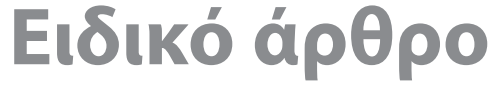 Special article
}

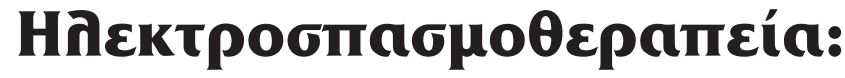 80 Xpóvia ecpappoyńs

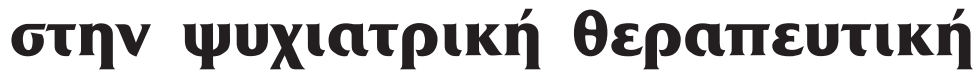

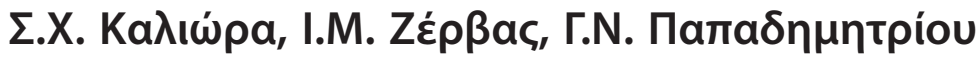

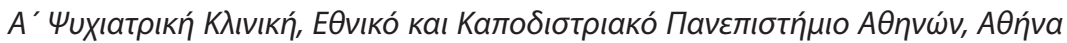

Uuxıatpıkń 2018, 29:291-302

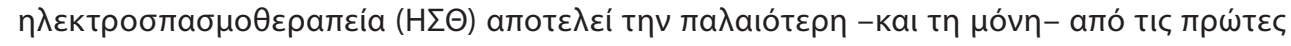

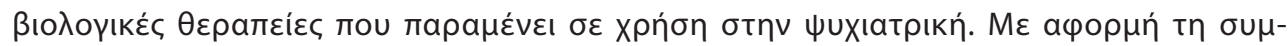

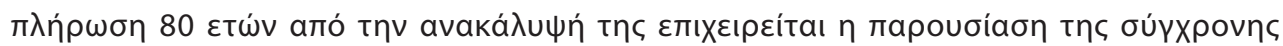

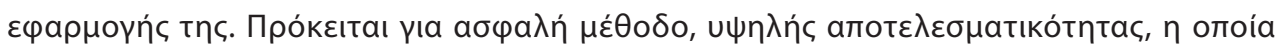

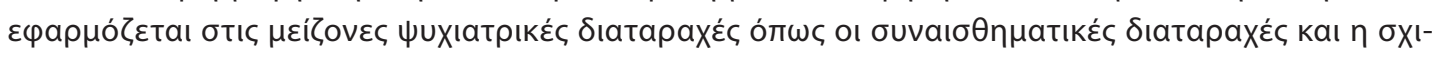

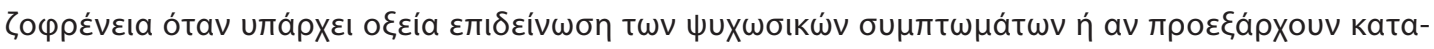

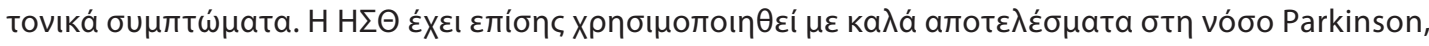

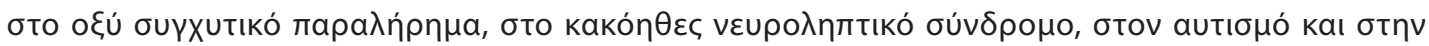

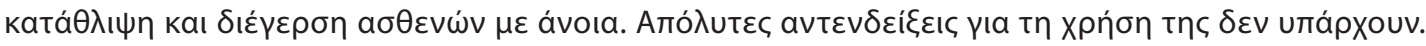

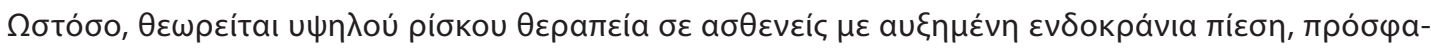

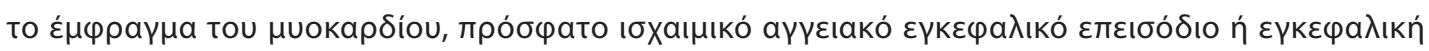

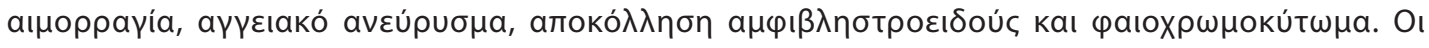

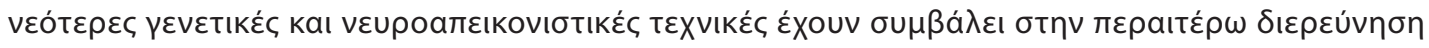

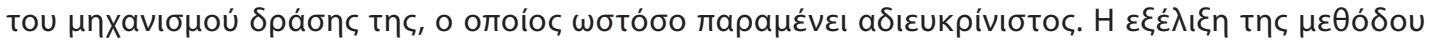

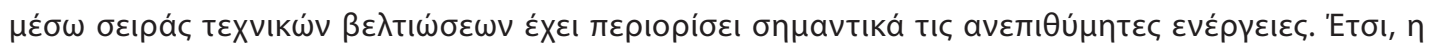

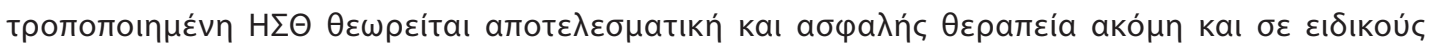

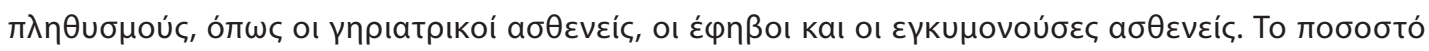

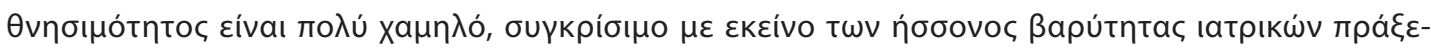

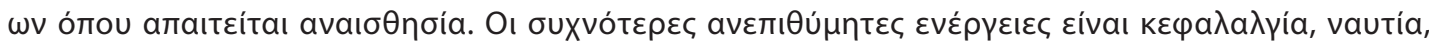

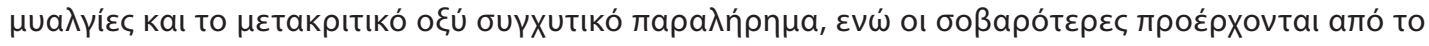

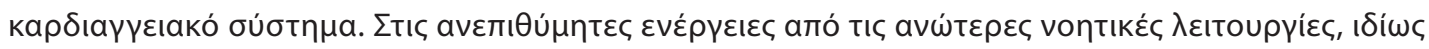




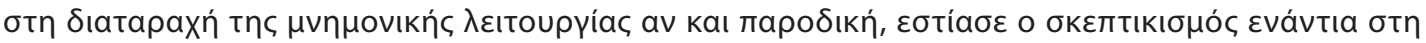

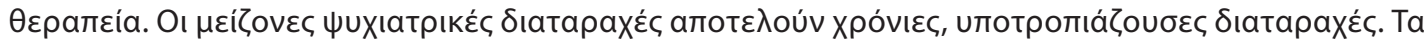

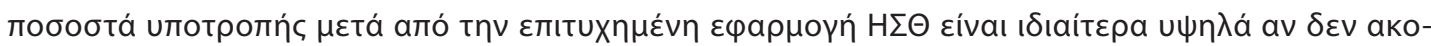

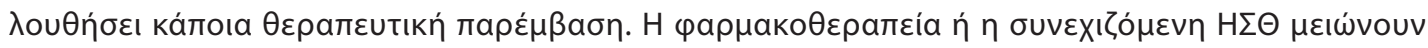

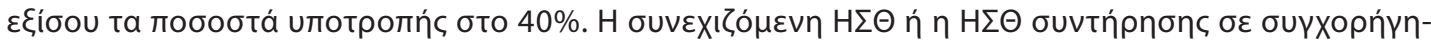

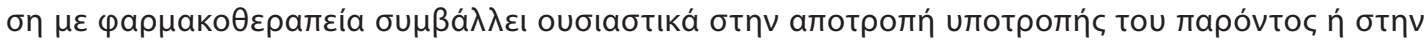

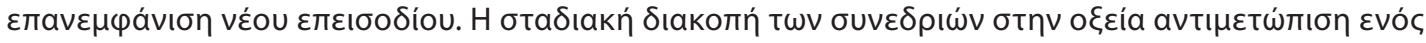

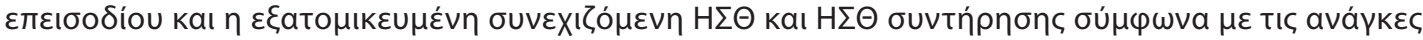

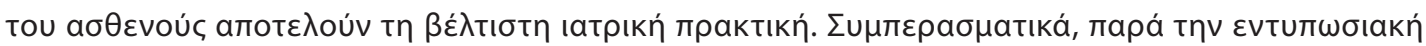

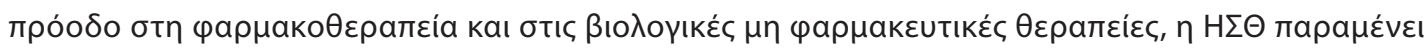

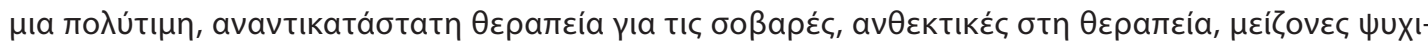

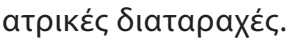

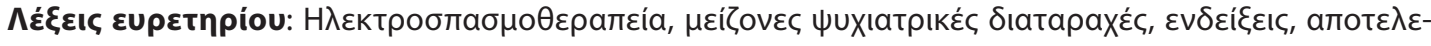
бнатіко́тпта.

\section{Eıбaywyń}

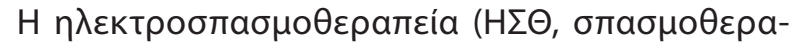

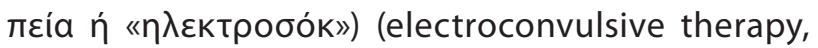

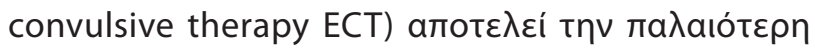

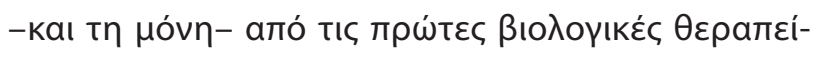

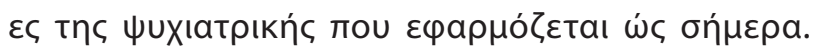

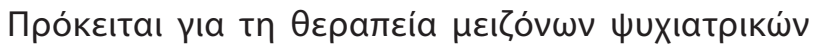

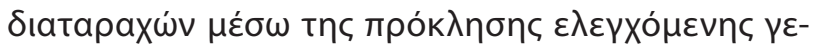

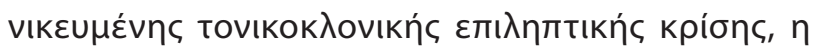

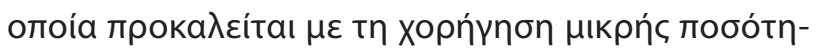

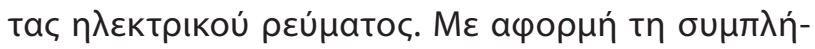

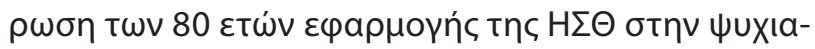

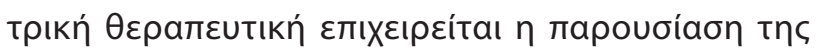

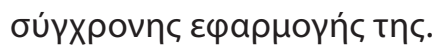

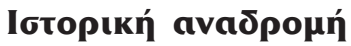

O Oúpypoc Ladislas Joseph Meduna (1896-1964)

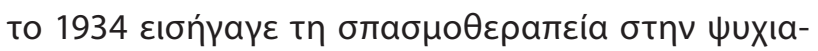

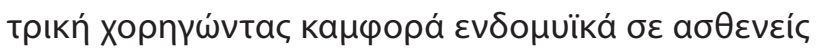

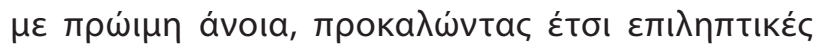

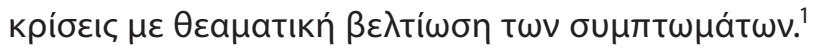

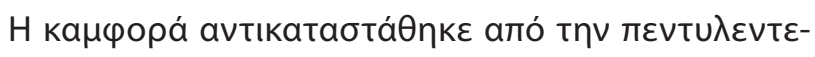

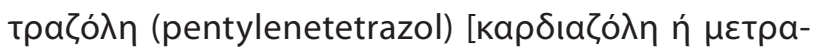

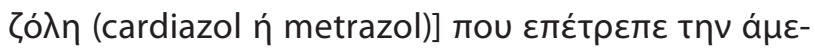

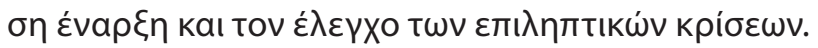

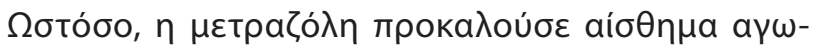

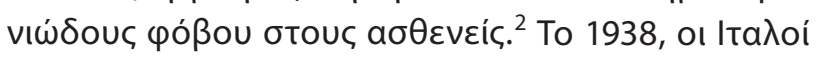
Ugo Cerletti (1877-1963) kaı Lucio Bini (1908-1964)

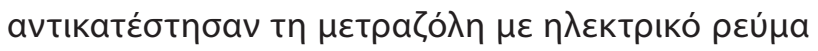

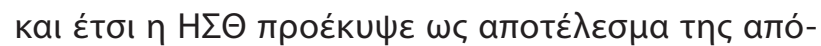

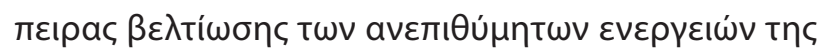

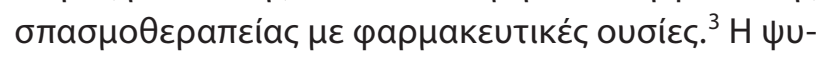

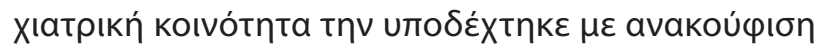

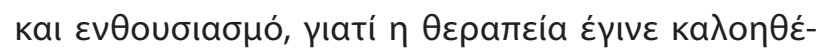

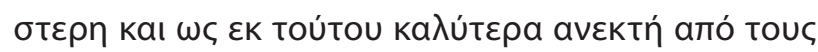

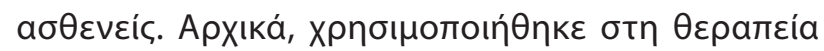

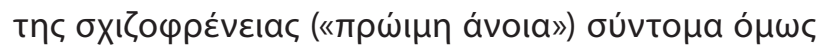

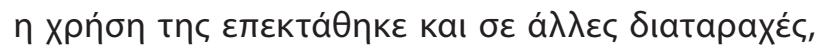

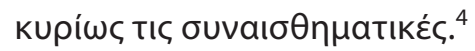

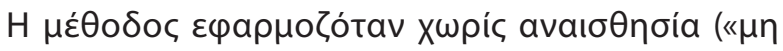

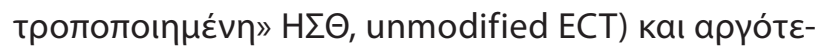

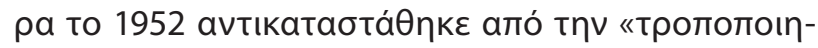

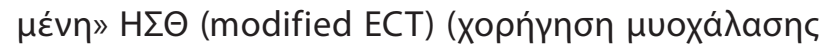

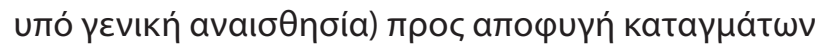

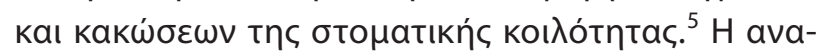

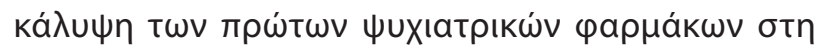

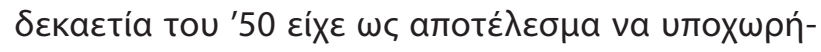

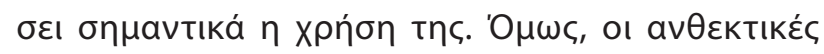

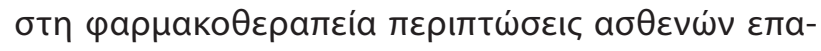

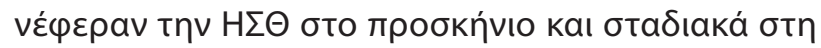

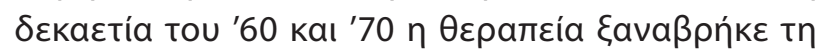

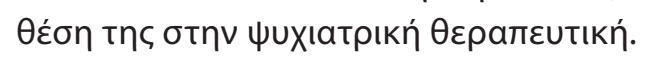

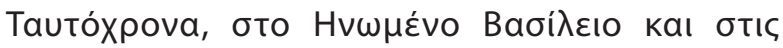

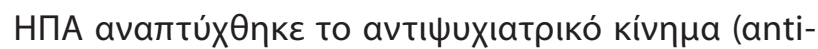

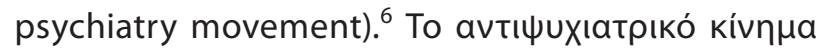

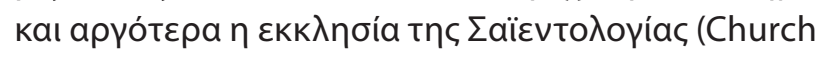

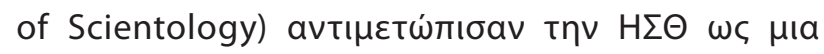

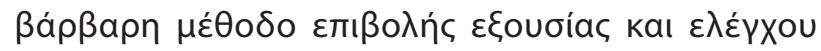

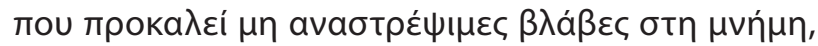




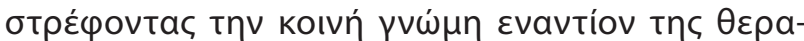

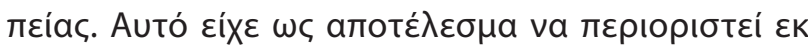

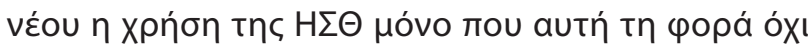

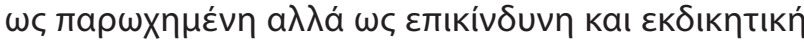

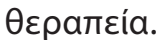

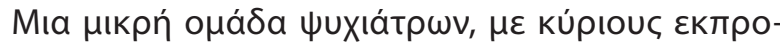

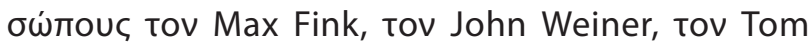

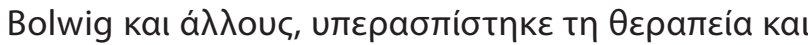

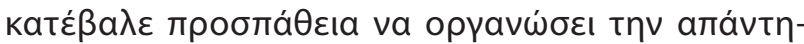

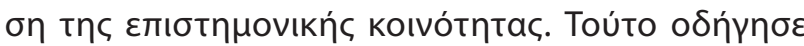

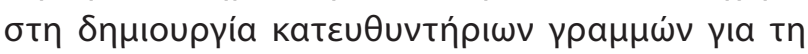

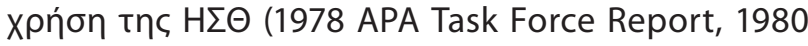
British Report, 1990, 2000 APA Task Force reports),

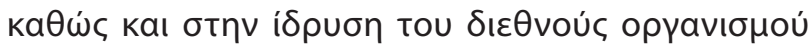
Association of Convulsive Therapy $\mu \varepsilon \dot{\varepsilon} \delta \rho a$ тІৎ HПA

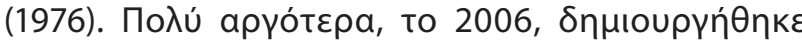

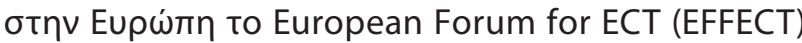

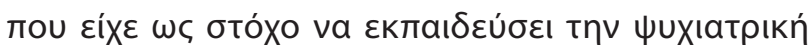

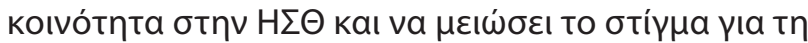

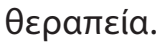

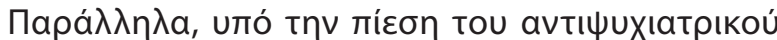

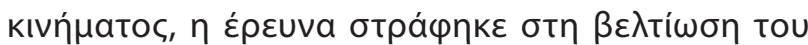

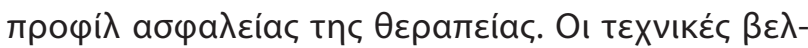

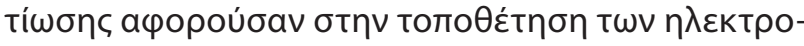

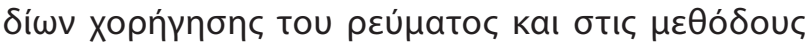

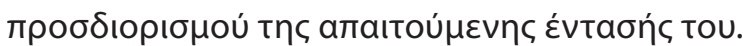

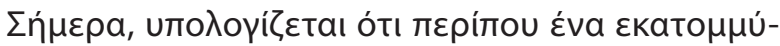

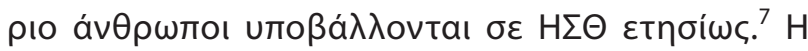

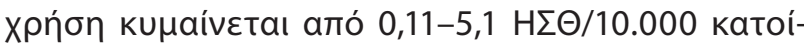

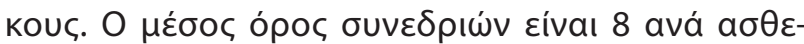

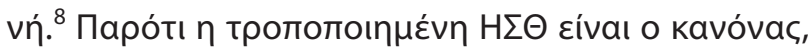
ако́

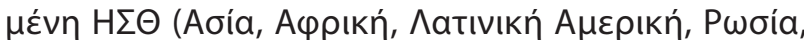

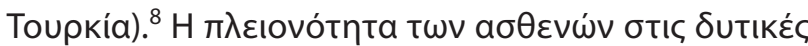

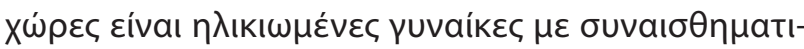

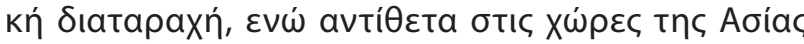

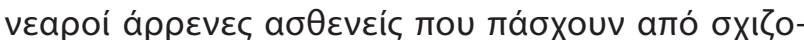

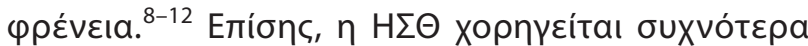

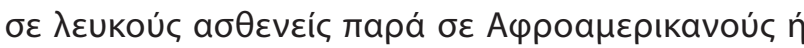

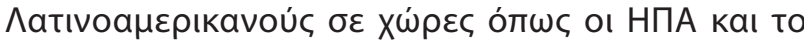

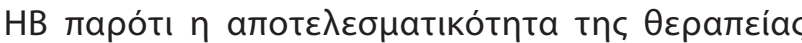

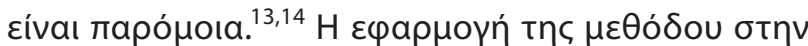

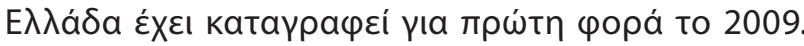

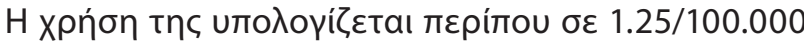

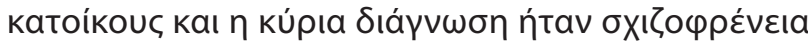

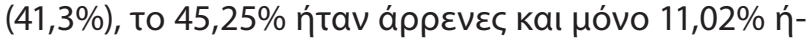

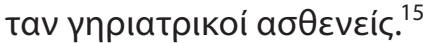

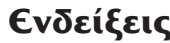

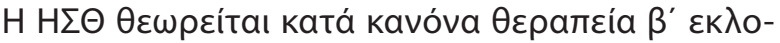

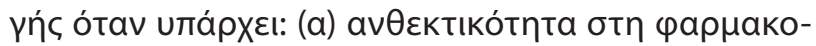

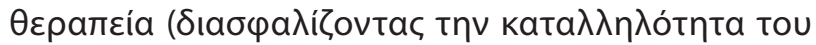

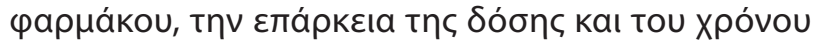

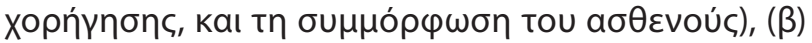

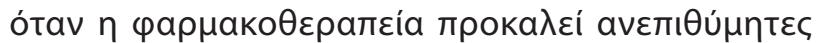

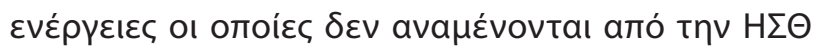

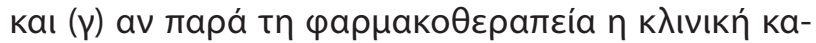

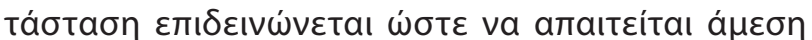

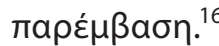

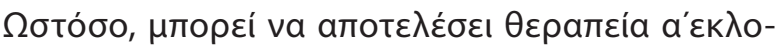

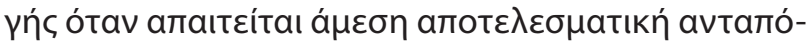

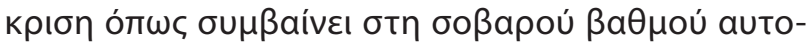

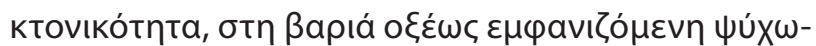

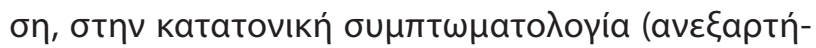

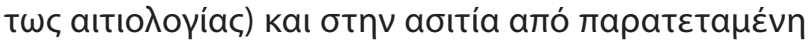

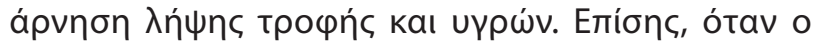

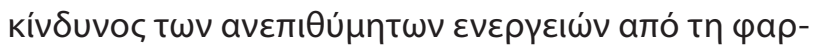

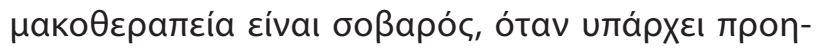

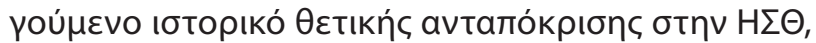

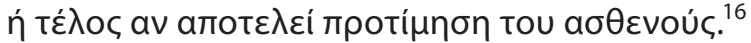

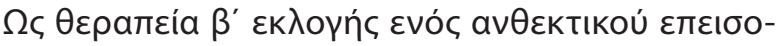

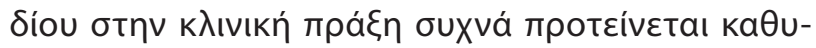

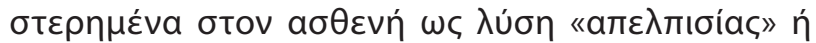

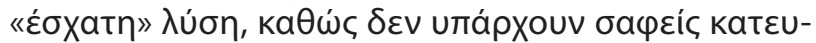

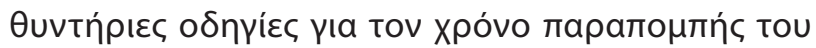

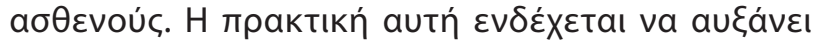

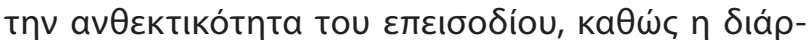

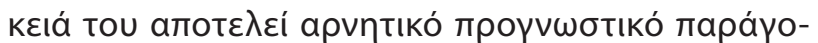

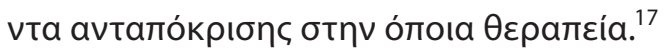

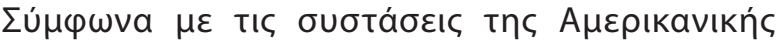

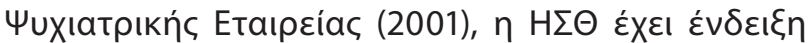

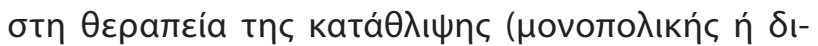

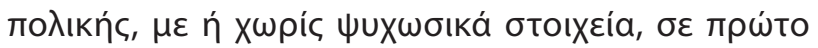

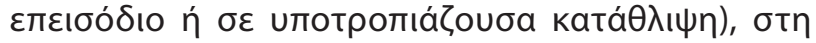

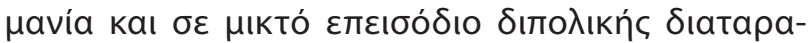

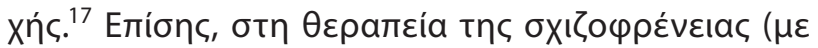

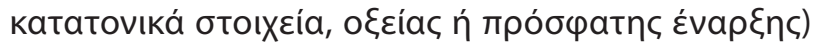

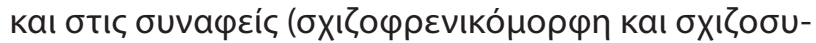

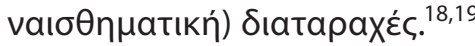




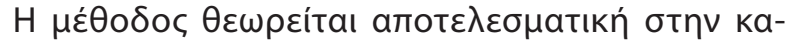

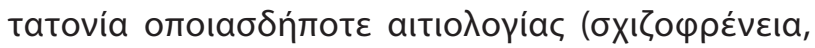

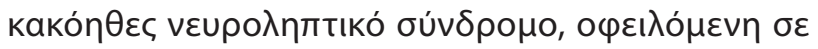

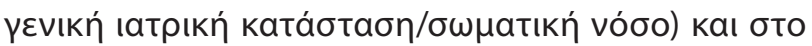

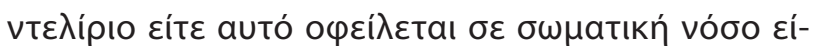

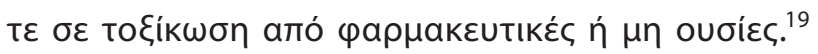

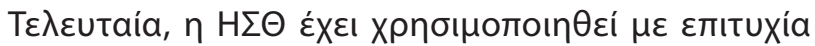

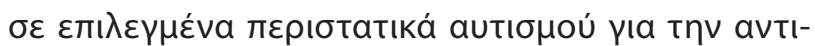

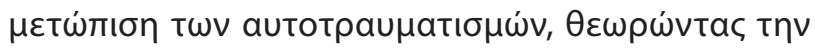

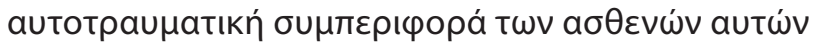

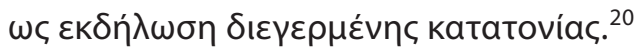

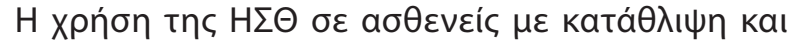

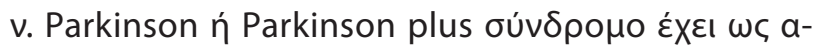

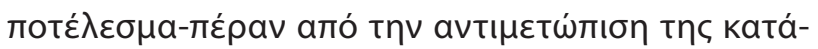

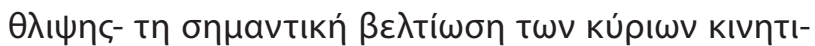

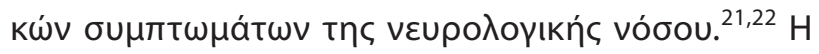

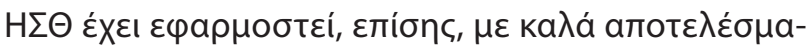

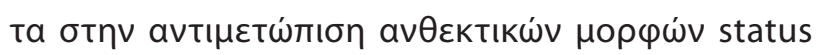
epilepticus. ${ }^{23,24}$

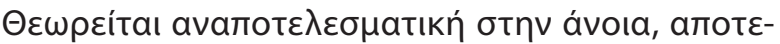

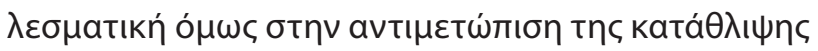

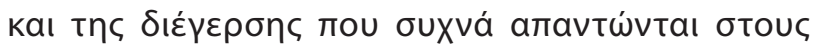

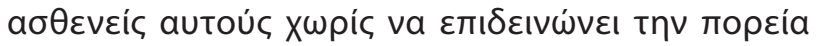

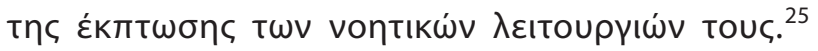

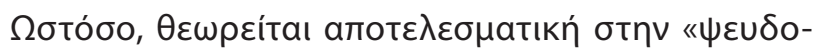

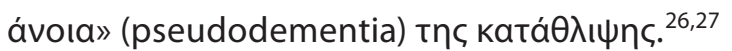

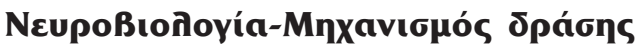

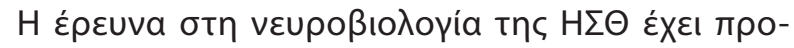

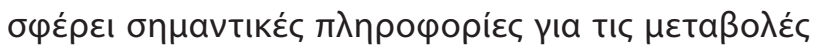

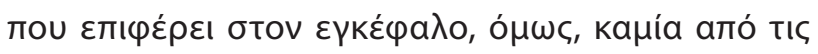

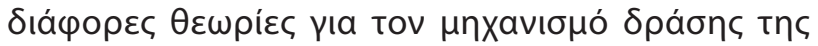

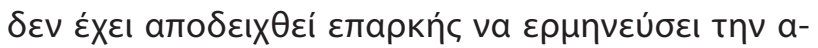

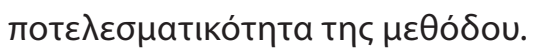

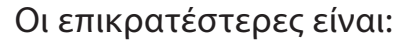

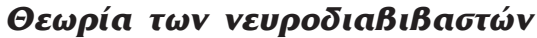

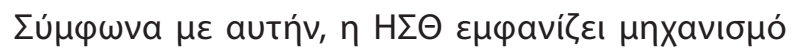

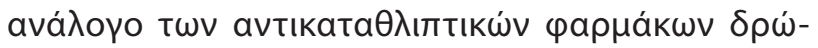

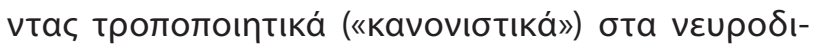

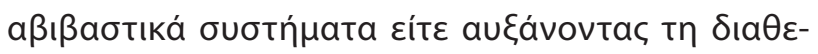

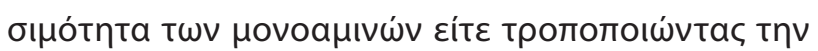

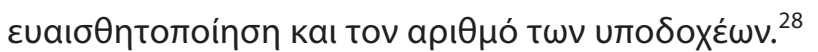

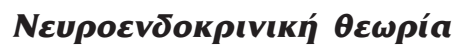

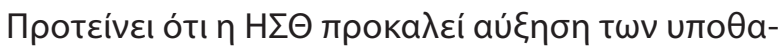

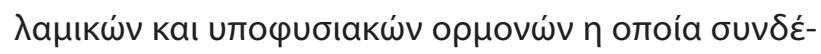

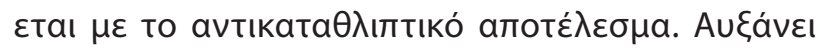

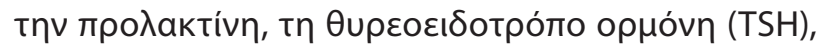

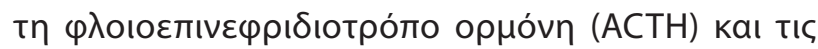

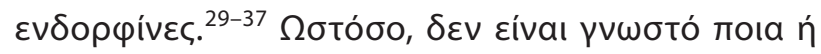

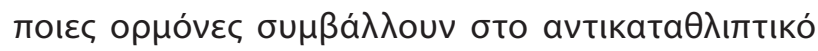

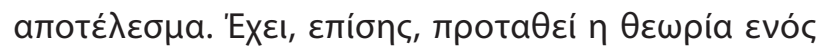
a

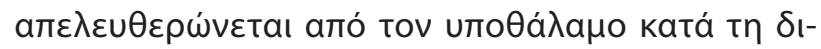

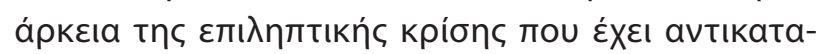

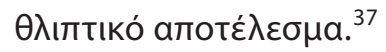

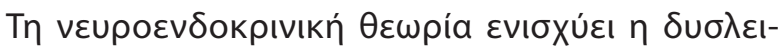

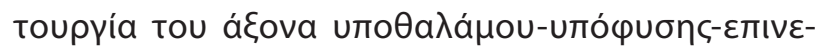

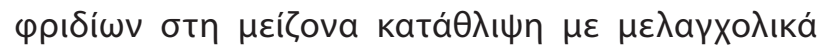

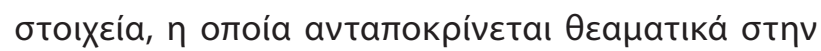
$\mathrm{H} \Sigma \Theta$.

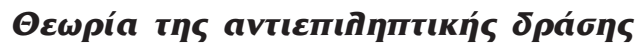

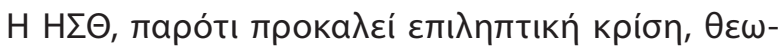

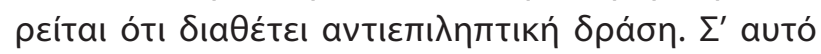

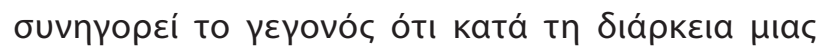

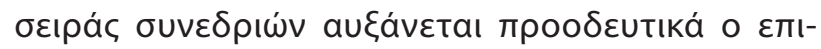

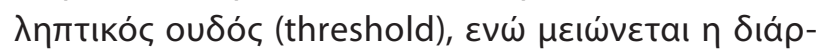

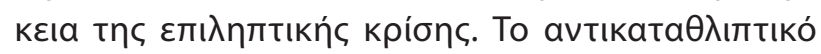

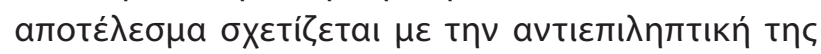

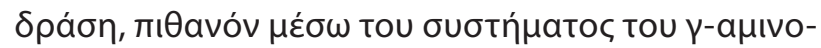
ßoutupıкоú o६źç ( $\gamma$-aminobutyric acid, GABA). ${ }^{38,39}$

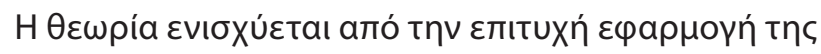

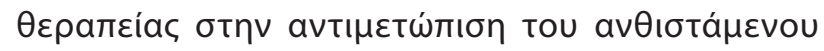
status epilepticus. ${ }^{40,41}$

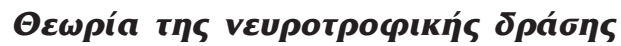

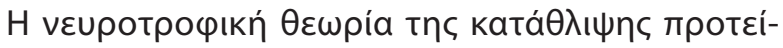

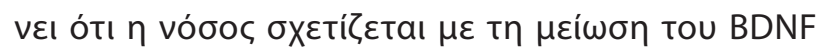

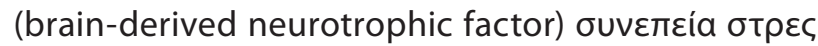

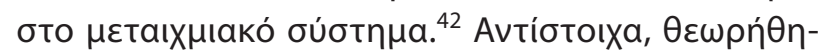

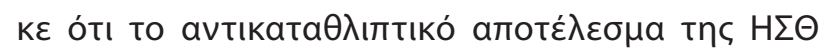

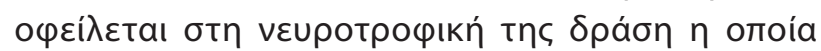

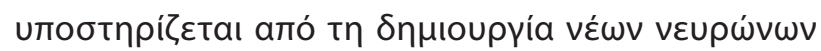

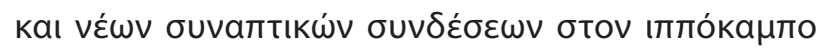

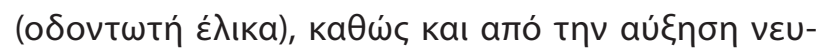

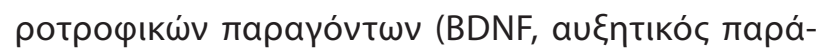

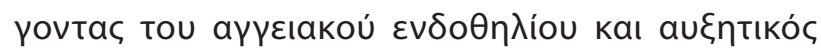

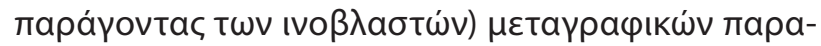




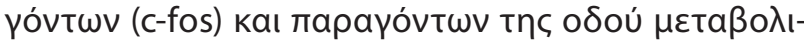

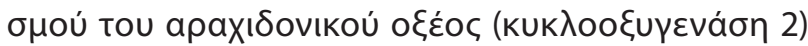

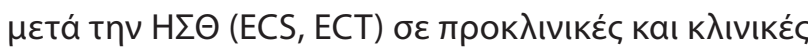
$\mu \varepsilon \lambda \varepsilon^{\prime} \varepsilon{ }^{4}{ }^{43-46}$

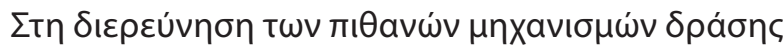

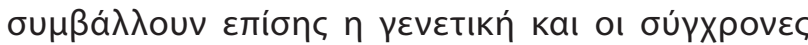

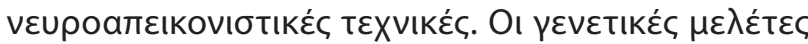

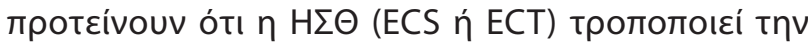

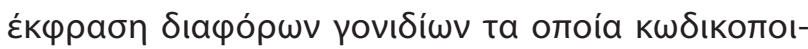

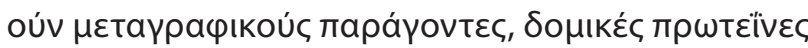

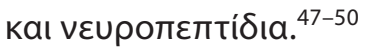

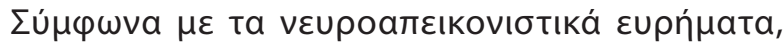

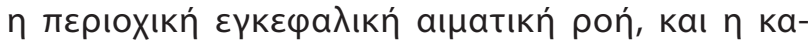

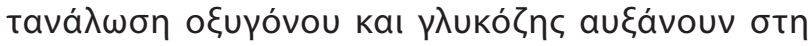

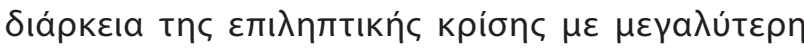

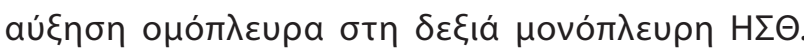

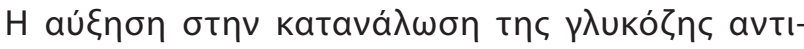

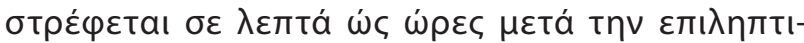

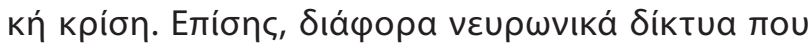

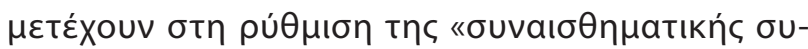

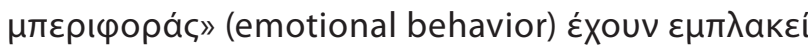

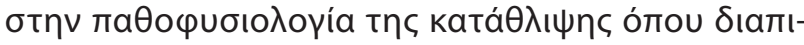

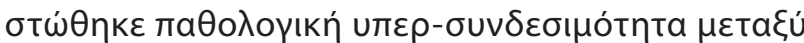

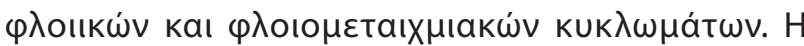

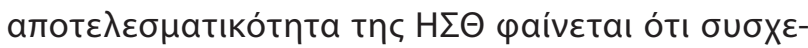

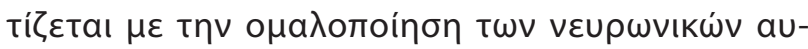

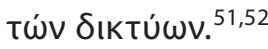

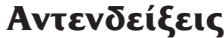

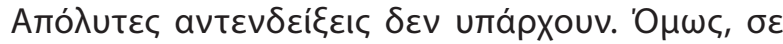

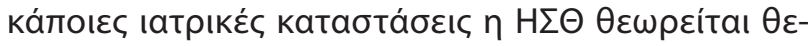

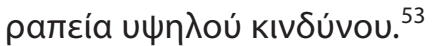

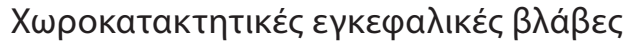

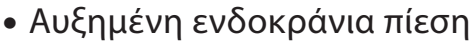

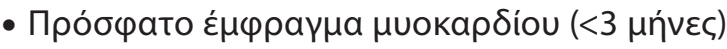

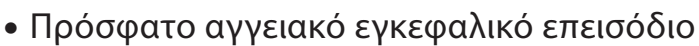

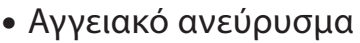

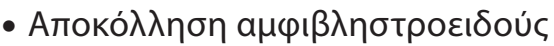

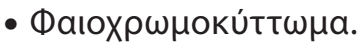

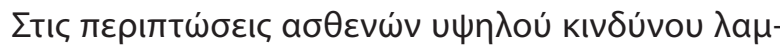

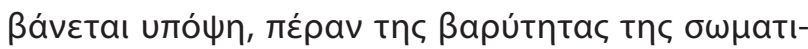

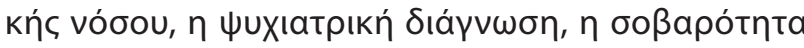

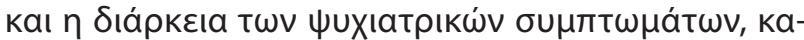

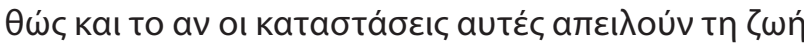

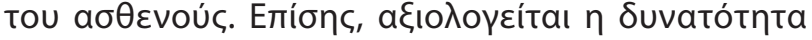

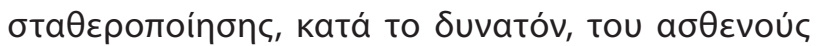

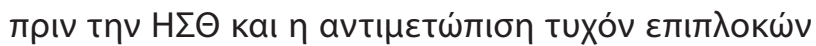

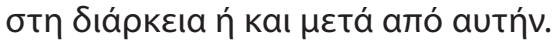

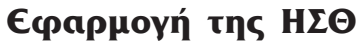

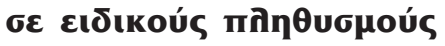

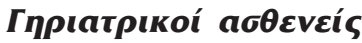

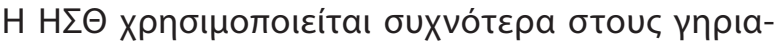

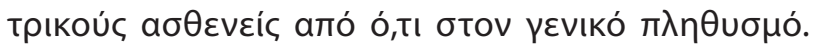

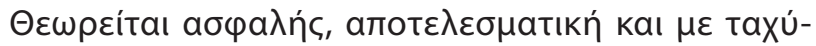

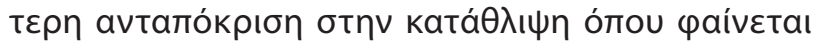

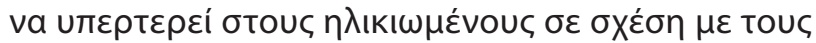

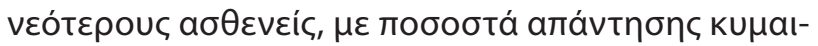

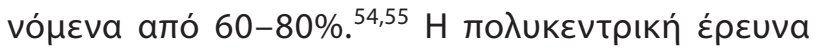

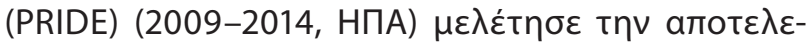

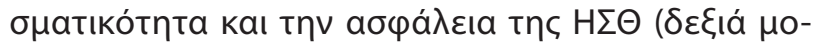

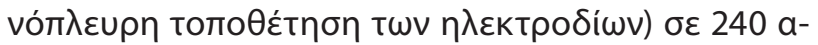

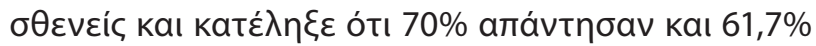

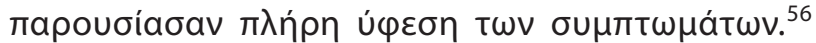

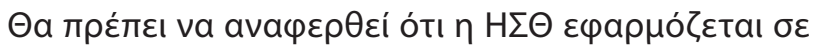

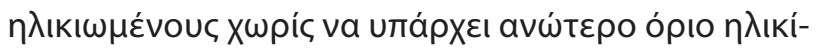

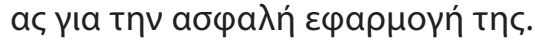

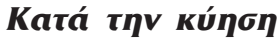

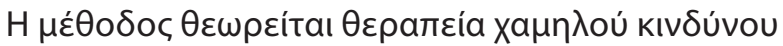

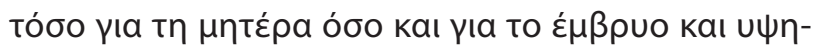

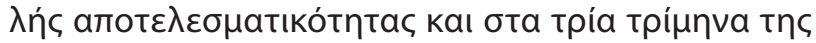

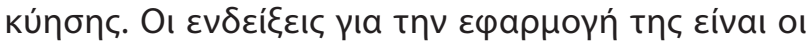

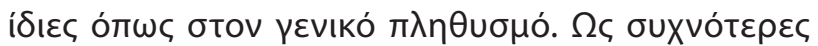

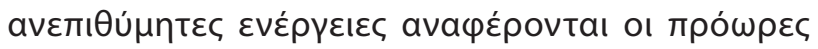

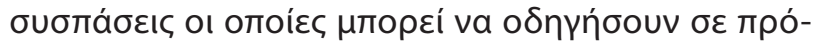

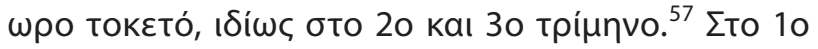

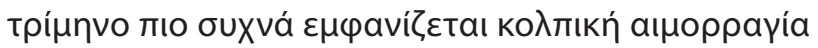

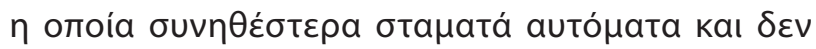

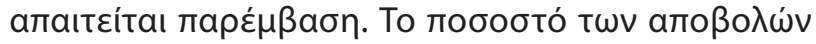

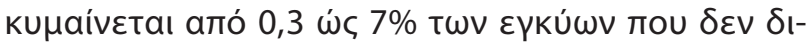

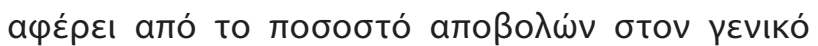

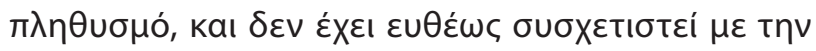

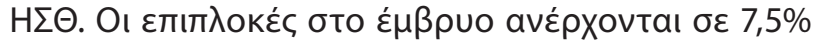

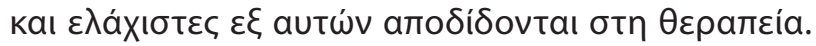

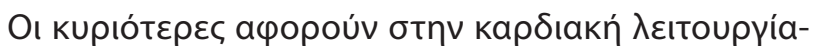

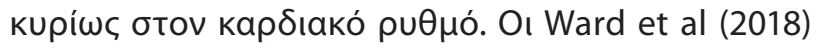

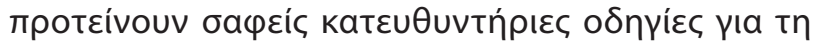

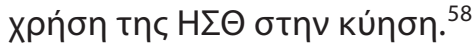




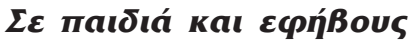

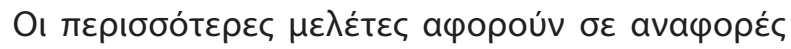

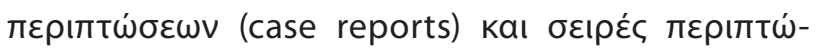

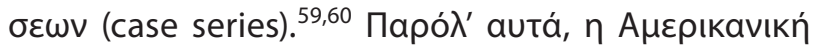

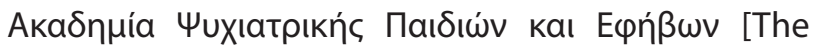
American Academy of Child and Adolescent Psychiatry

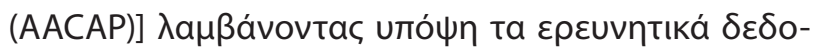

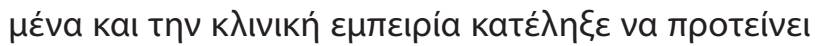

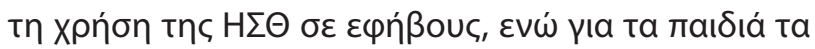

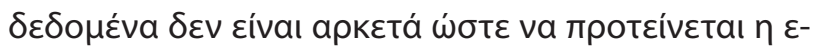

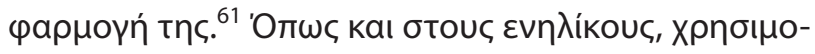

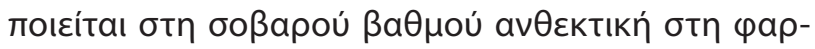

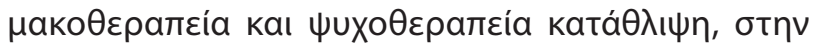

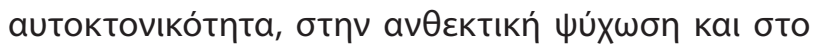

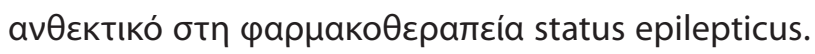

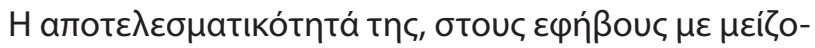

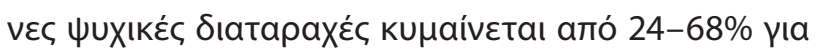

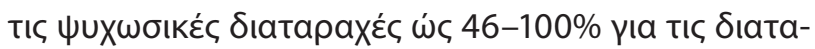

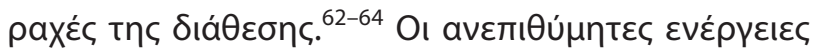

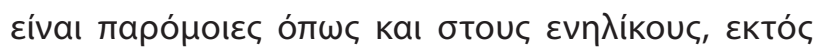

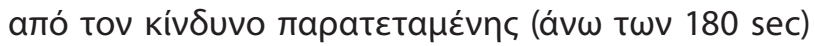

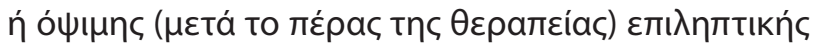

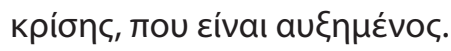

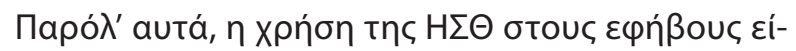

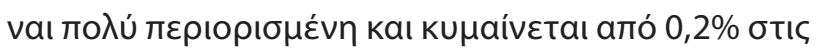

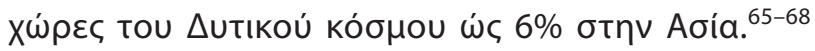

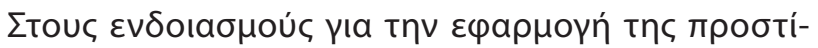

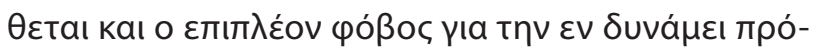

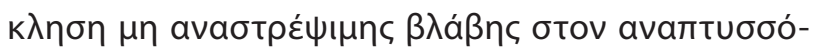

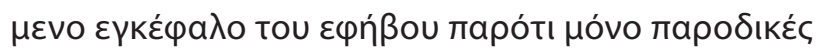

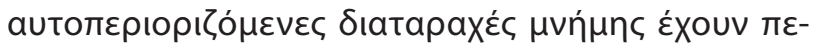
рıррачعí. ${ }^{69,70}$

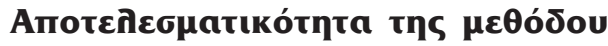

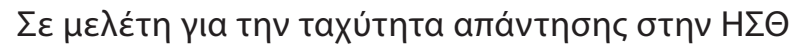

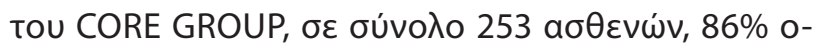

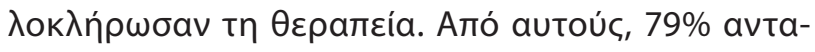

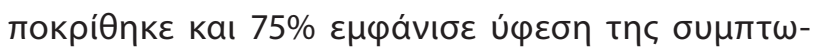

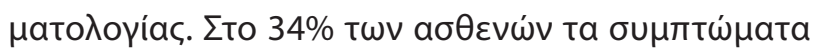

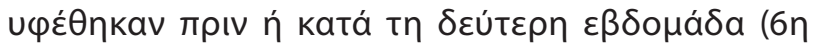

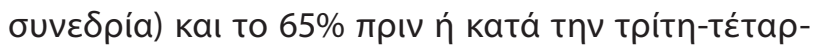

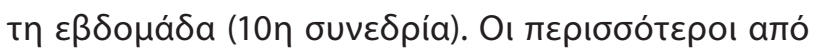

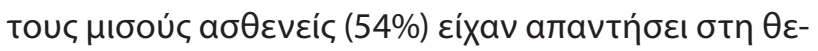

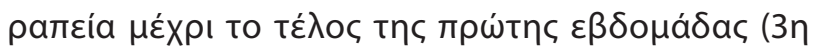

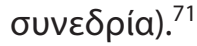

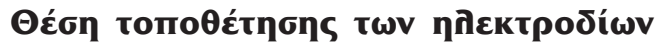

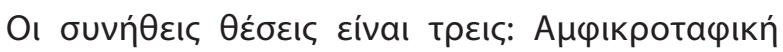

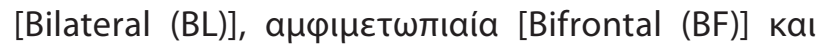

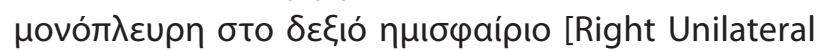
(RUL)].

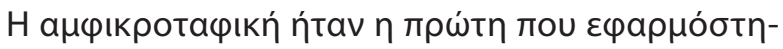

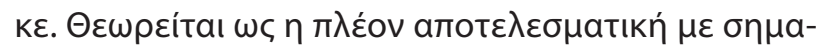

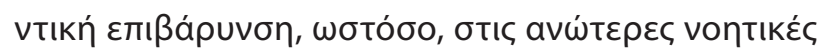

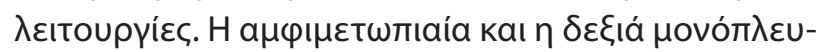

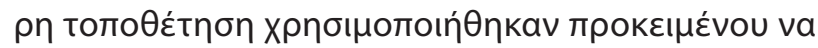

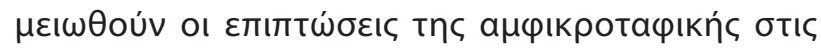

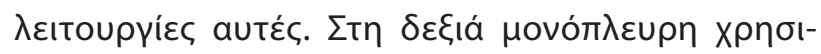

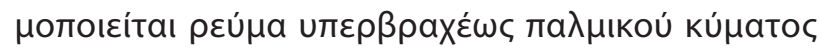

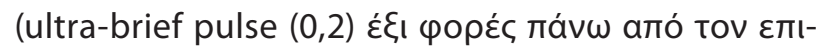

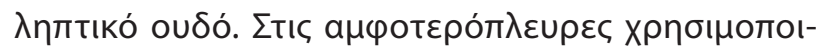

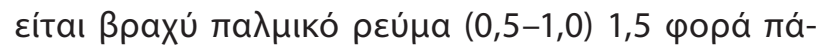

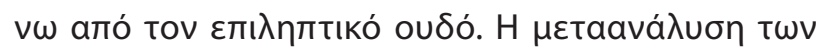

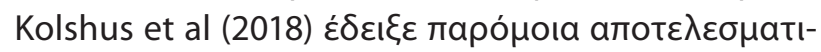

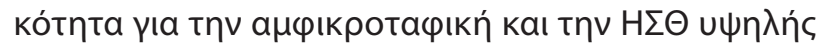

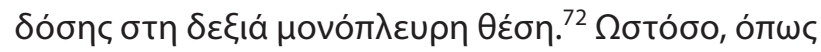

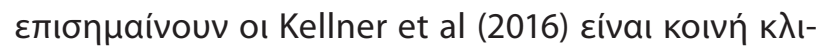

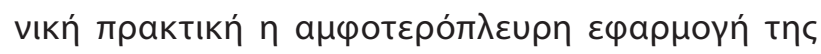

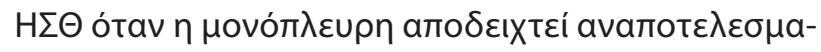
тIKń. ${ }^{73,74}$

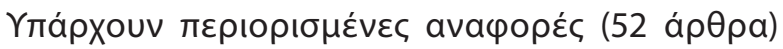

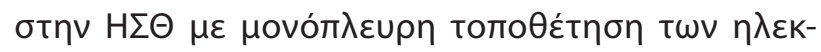

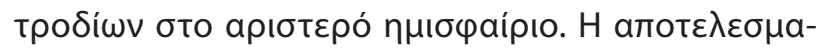

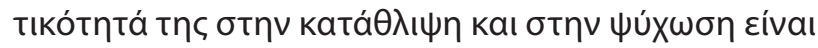

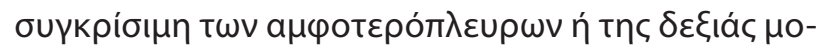

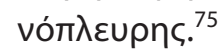

\section{Avaıonoía}

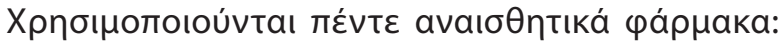

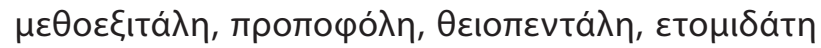

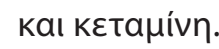

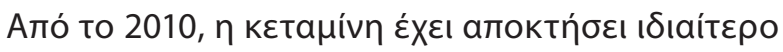

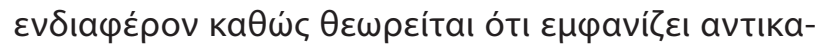

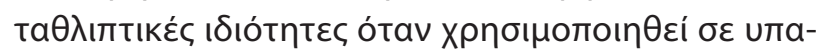

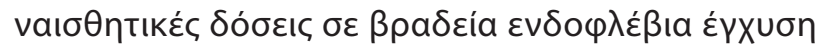

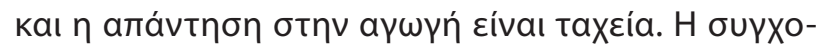

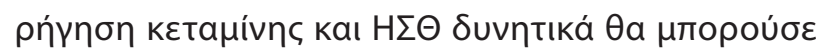

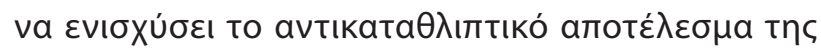

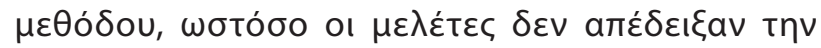

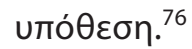




\section{Muoxáâan}

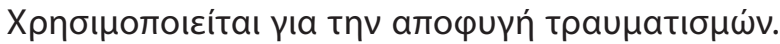

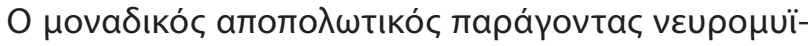

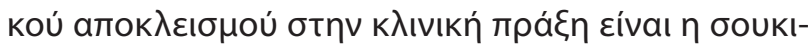

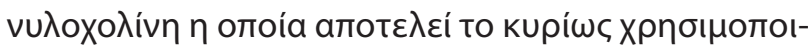

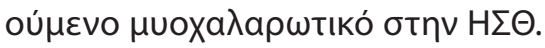

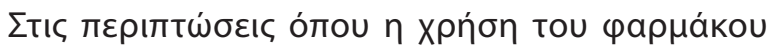

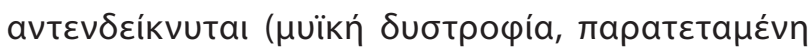

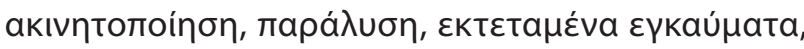

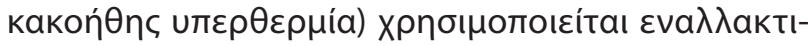

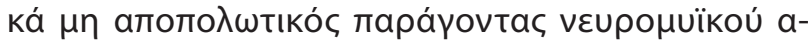

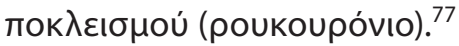

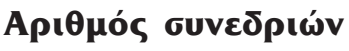

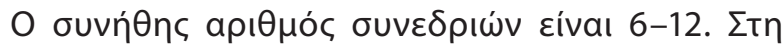

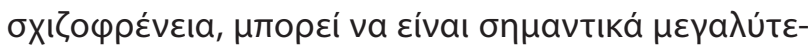

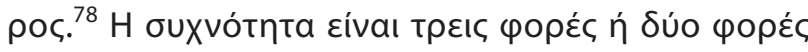

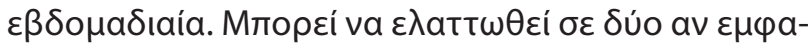

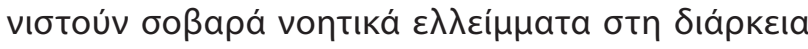

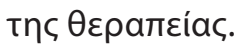

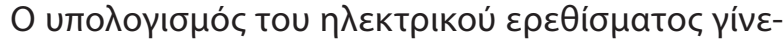

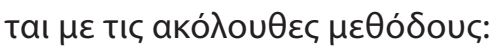

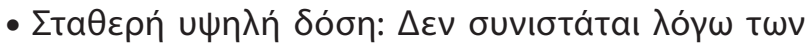

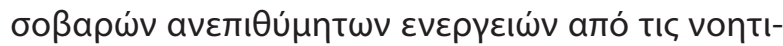

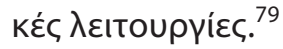

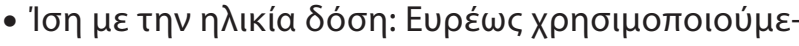

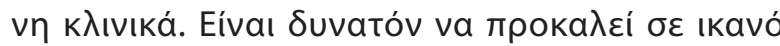

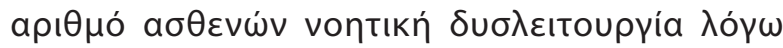

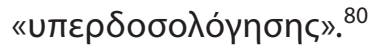

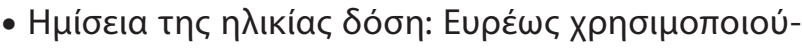

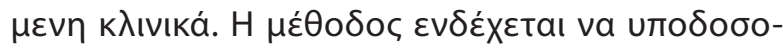

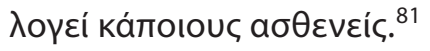

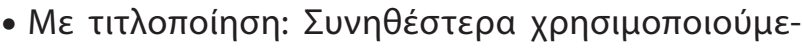

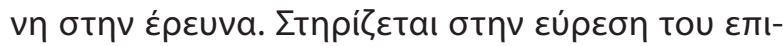

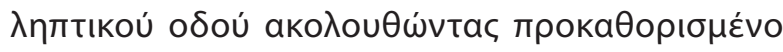

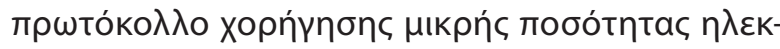

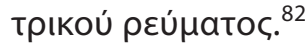

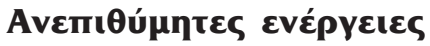

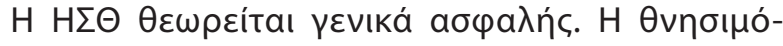

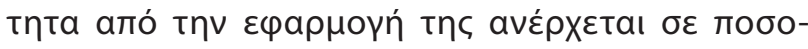

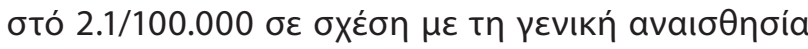
$(3.4 / 100.000){ }^{83}$

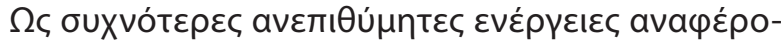

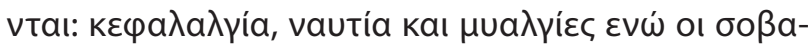

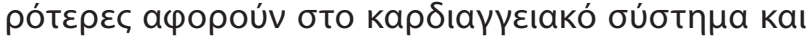

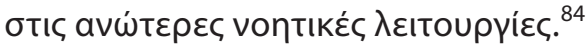

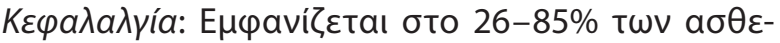

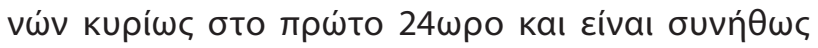

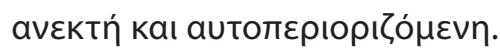

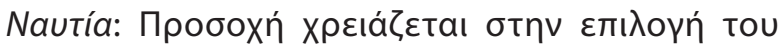

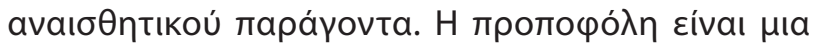

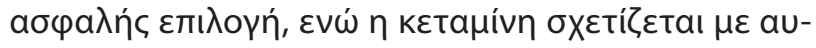

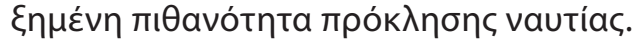

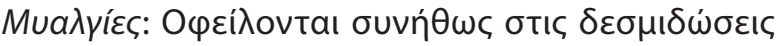

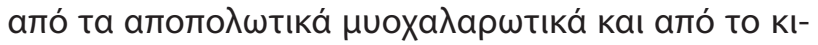

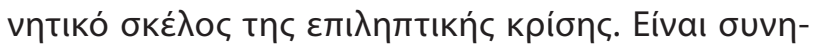

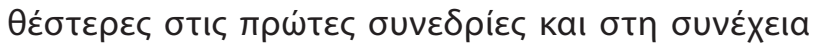
Uтохшроúv.

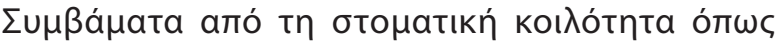

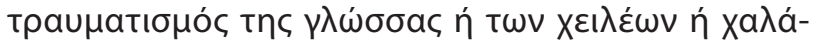

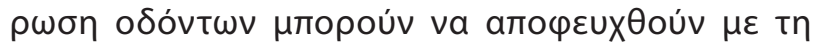

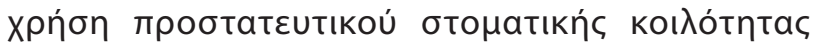

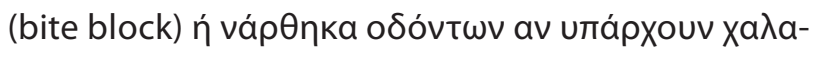

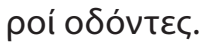

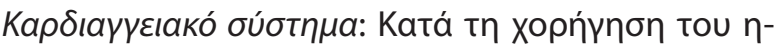

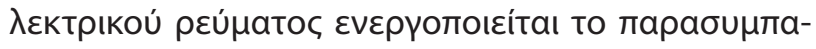

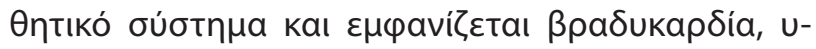

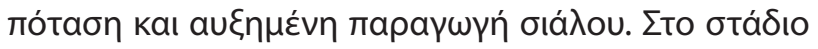

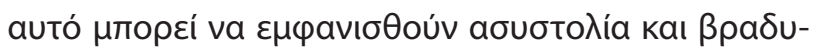

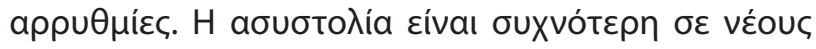

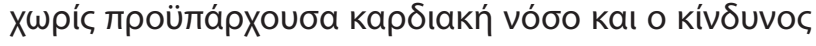

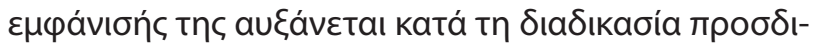

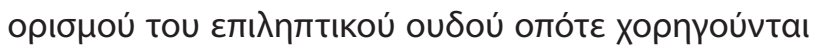

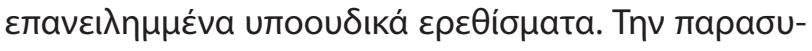

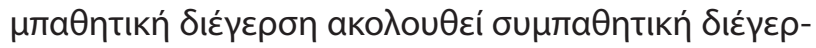

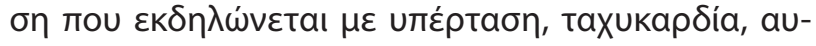

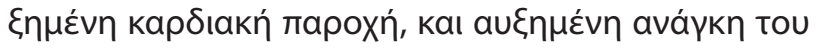

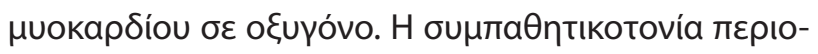

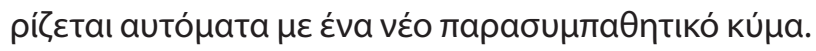

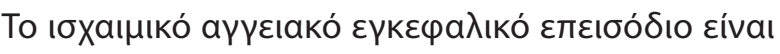
бтávı

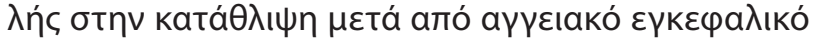

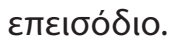

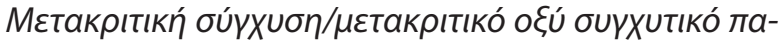

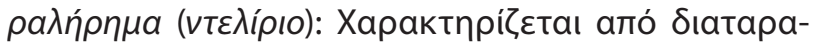

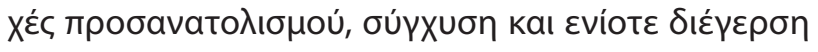

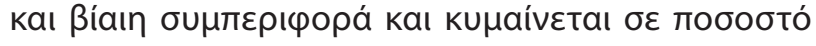

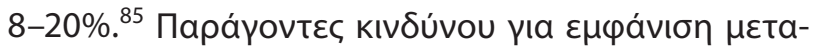

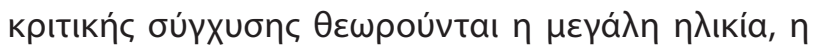




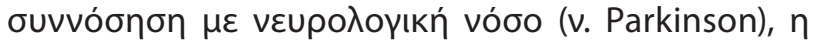

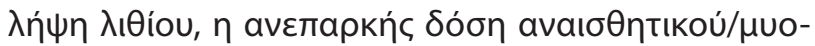

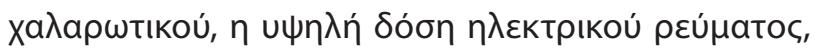

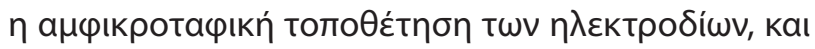

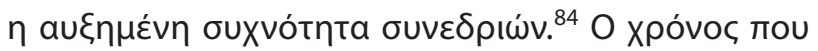
a

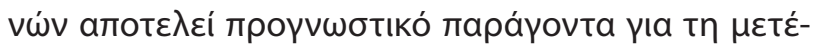

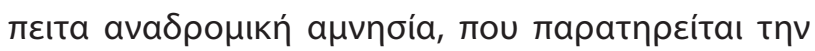

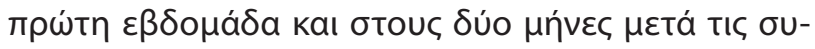

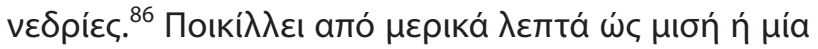

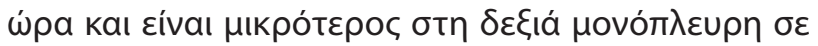

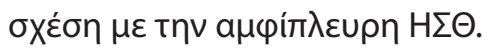

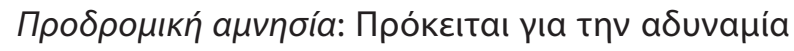

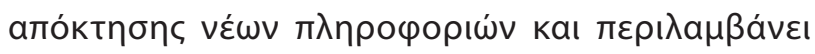

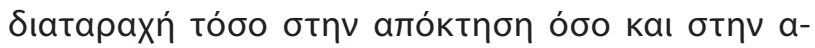

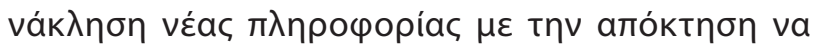

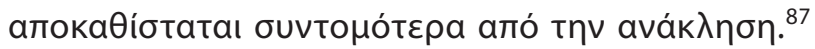

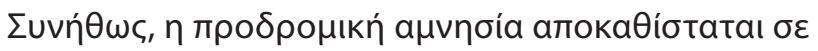

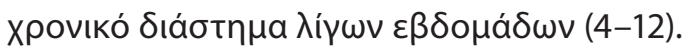

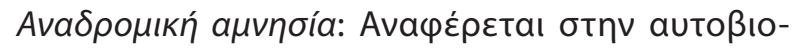

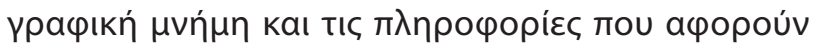

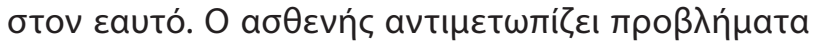

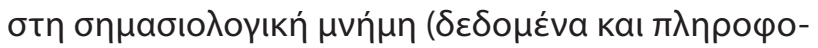

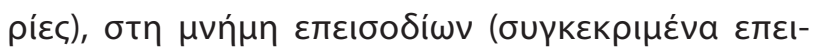

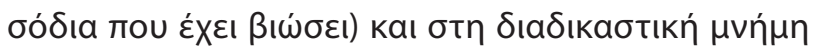

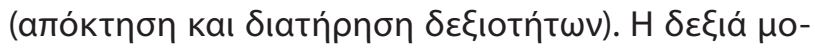

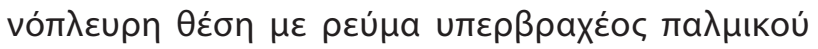

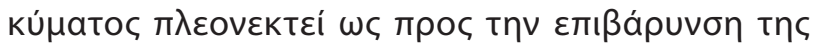

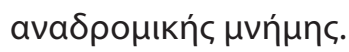

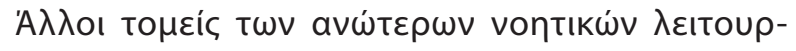

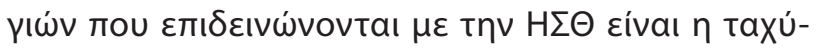

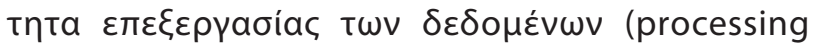

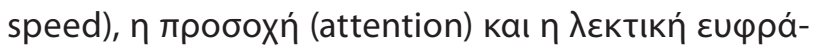
$\delta \varepsilon ı$ (verbal fluency). ${ }^{84}$

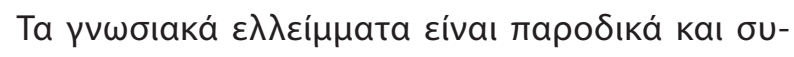

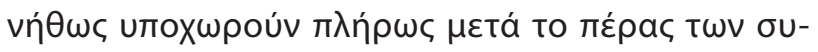

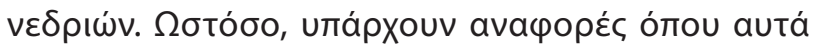

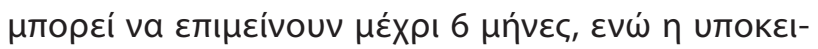

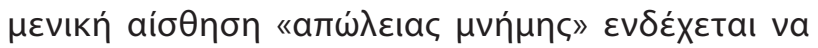
пapateíveta.. ${ }^{84}$

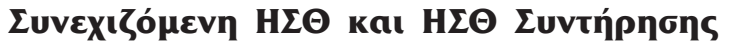

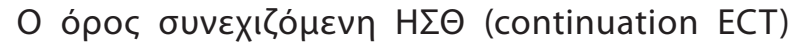

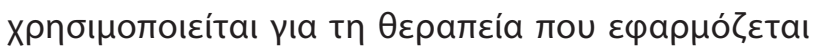

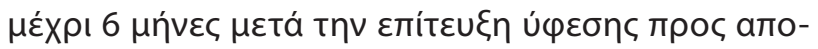

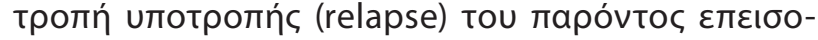

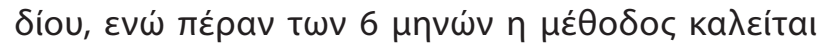

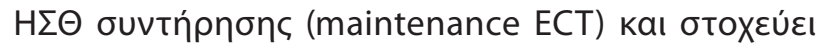

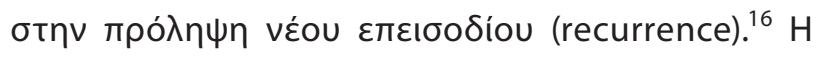

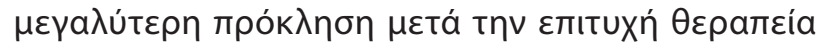

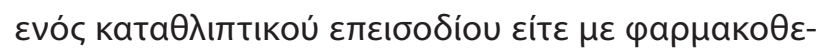

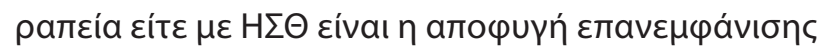

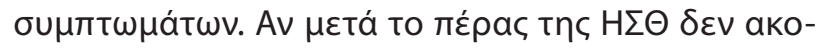

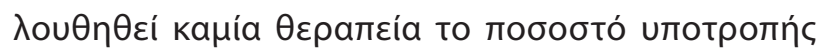

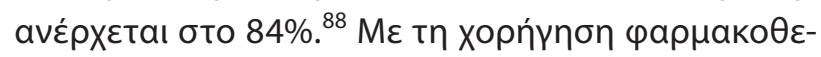

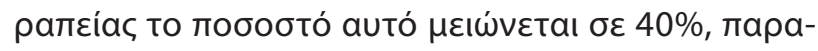

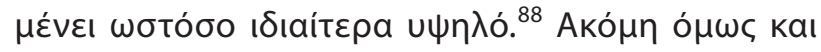

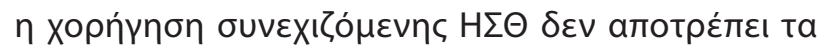

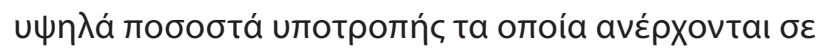

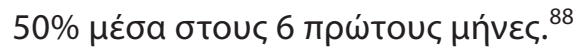

To Consortium for Research in ECT group (CORE

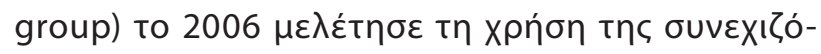

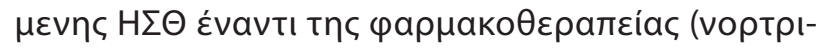

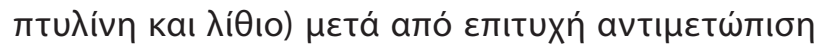

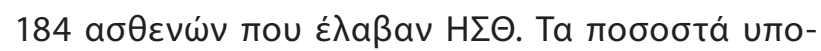

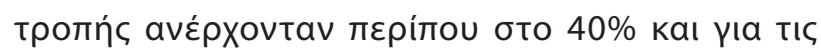

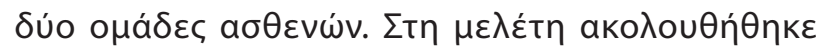

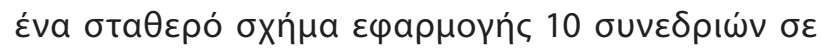

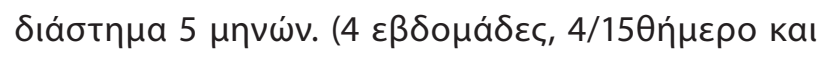

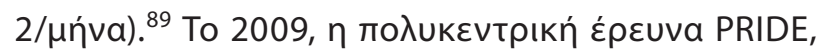

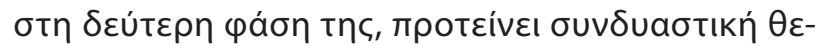

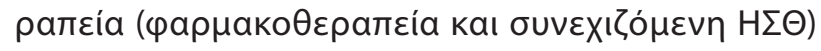

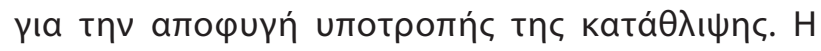

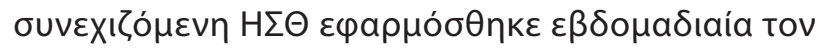

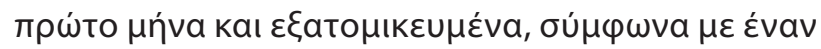

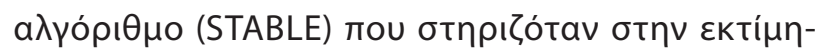

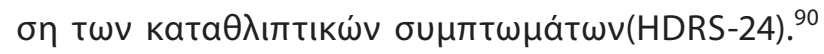

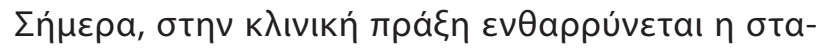

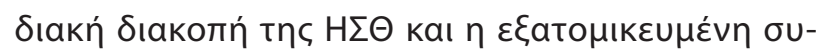

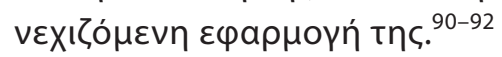

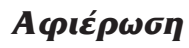

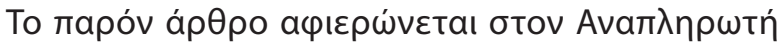

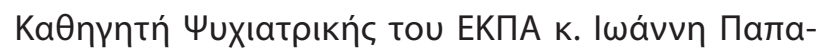

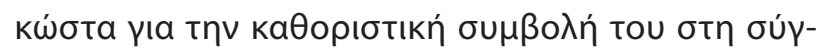

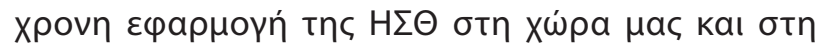

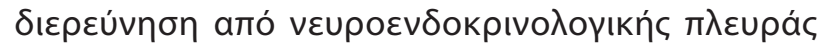

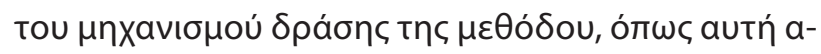

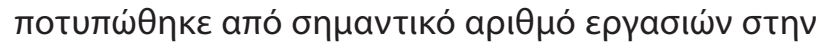

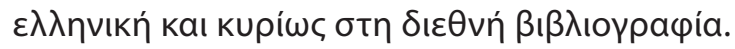




\title{
Electroconvulsive therapy: 80 years of use in psychiatry
}

\author{
S.C. Kaliora, I.M. Zervas, G.N. Papadimitriou \\ 1st Department of Psychiatry, National and Kapodistrian University of Athens, Athens, Greece
}

Psychiatriki 2018, 29:291-302

Electroconvulsive therapy (ECT) is the oldest among the early biological treatments introduced in psychiatry, and the only one still in use. In this paper we attempt a brief presentation of ECT usage over the last 80 years, since it was originally introduced. It is a safe, well-tolerated, and highly effective treatment option for major psychiatric disorders, such as mood disorders and schizophrenia, especially when there is an acute exacerbation of psychotic symptoms or if catatonic symptoms are prominent. ECT has also been used successfully for the treatment of Parkinson's disease, delirium, neuroleptic malignant syndrome, autism and agitation and depression in demented patients. There are no absolute contraindications. However, it is considered a high risk procedure for patients with increased intracranial pressure, recent myocardial infarction, recent cerebral hemorrhage or stroke, vascular aneurysm, retinal detachment and pheocromocytoma. Modern genetic and neuroimaging techniques have helped clarify possible mechanisms of action of ECT, but much remains unknown. Improvement of this method through a number of technical advancements has contributed in the reduction of side effects. Thus, modified ECT is currently considered as an effective and safe form of treatment even in vulnerable populations such as the geriatric patients, the adolescents and the pregnant patients. The mortality rate is very low, comparable to that of a minor anesthetic procedure. The most common adverse events are headache, nausea, myalgias and postictal delirium while the most severe are the cardiovascular side effects. Of note, the cognitive side effects especially amnesia, although transient, has been the focus of skepticism against the treatment. Major psychiatric disorders are chronic, recurring disorders. The relapse rate after a successful course of ECT without any intervention is extremely high. Pharmacotherapy or continuation ECT reduces equally the relapse rate up to $40 \%$. Continuation and maintenance ECT, in combination with pharmacotherapy, have been successfully used in preventing relapse and recurrence. Gradual tapering off acute ECT treatments and individualized continuation and maintenance ECT treatments based on the needs of each patient seems the optimum clinical practice. Conclusively, despite impressive new developments in pharmacotherapy and in biological non pharmacological treatments ECT remains a valuable, irreplaceable treatment option for debilitating, resistant major psychiatric disorders.

Key words: Electroconvulsive therapy, major psychiatric disorders, indications, efficacy.

\section{Bıßntoypacpía}

1. Gazdag G, Bitter I, Ungvari GS, Baran B, Fink M. László Meduna's pilot studies with camphor inductions of seizures: the first 11 patients. J ECT 2009, 25:3-11, doi: 10.1097/YCT. 0b013e31819359fc

2. Cooper K, Fink M. The chemical induction of seizures in psychiatric therapy: were flurothyl (indoklon) and pentylenetetrazol (metrazol) abandoned prematurely? J Clin Psychopharmacol. 2014, 34:602-607, doi: 10.1097/JCP.0000000000000173

3. Cerletti, U, and L. Bini. 1938. Un nuovo metodo di shockterapie: 'l' elettroshock' (riassunto). Reale Accademia Medica
(Communicazione alla seduta del 28 maggio 1938-XVI della Reale Accademia Medica di Roma), Rome

4. Fink M. Electroshock: restoring the mind. New York, Oxford University Press, 1999

5. Holmberg G, Thesleff S. Succinyl-choline-iodide as a muscular relaxant in electroshock therapy. Am J Psychiatry 1952, 108:842-846, PMID: 14923897

6. Lebensohn ZM. The history of electroconvulsive therapy in the United States and its place in American psychiatry: a personal memoir. Compr Psychiatry 1999, 40:173-181, PMID: 10360611 
7. Prudic J, Olfson M, Sackeim HA. Electroconvulsive therapy practices in the community. Psychol Med 2001, 31:929-934, PMID: 11459391

8. Leiknes KA, Jarosh-von Schweder L, Høie B. Contemporary use and practice of electroconvulsive therapy worldwide. Brain Behav 2012, 2:283-344, doi: 10.1002/brb3.37

9. Reid WH, Keller S, Leatherman M, Mason M. ECT in Texas: 19 months of mandatory reporting. J Clin Psychiatry 1998, 59:8-13, PMID: 9491059

10. Glen T, Scott Al. Rates of electroconvulsive therapy use in Edinburgh (1992-1997). J Affect Disord 1999, 54:81-85, PMID: 10403150

11. Moksnes KM, Vatnaland T, Eri B, Torvik NH. Electroconvulsive therapy in the Ullevaal region of Oslo 1988-2002. Tidsskr Nor Laegeforen 2006, 126:1750-1753, PMID:16794670

12. Chanpattana W, Kramer BA, Kunigiri G, Gangadhar BN, Kitphati $\mathrm{R}$, Andrade C. A survey of the practice of electroconvulsive therapy in Asia. J ECT 2010, 26:5-10, doi: 10.1097/YCT. 0b013e3181a74368

13. Williams J, Chiu L, Livingston R A. Electroconvulsive Therapy (ECT) and Race: A Report of ECT Use and Sociodemographic Trends in Texas. J ECT 2017, 33:111-116, doi: 10.1097/YCT. 0000000000000379

14. Euba R. Electroconvulsive therapy and ethnicity. J ECT 2012, 28:24-26, doi: 10.1097/YCT.0b013e318223834e

15. Kaliora SC, Braga RJ, Petrides G, Chatzimanolis J, Papadimitriou GN, Zervas IM. The practice of electroconvulsive therapy in Greece. J ECT 2013, 29:219-224, doi: 10.1097/YCT. 0b013e $31827 \mathrm{e} 0 \mathrm{~d} 49$

16. American Psychiatric Association Committee on Electroconvulsive Therapy The Practice of Electroconvulsive Therapy: Recommendations for treatment, training and privileging (A Task Force Report of The American Psychiatric Association), American Psychiatric Pub, 2001

17. Haq AU Sitzmann AF, Goldman ML, Maixner DF, Mickey BJ. Response of depression to electroconvulsive therapy: a metaanalysis of clinical predictors. J Clin Psychiatry 2015, 76:13741384, doi: 10.4088/JCP.14r09528

18. National Institute for Health and Clinical Excellence. NICE technology appraisal guidance No. 59: The clinical effectiveness and cost effectiveness of Electroconvulsive Therapy (ECT) for depressive illness, schizophrenia, catatonia and mania. 2003, Available at: http//www.nice.org.uk/guidance/ta59

19. APA's Diagnostic and Statistical Manual of Mental Disorders, Edition IV, American Psychiatric Publishing, Washington, DC, 1994

20. Wachtel LE, Shorter E, Fink M. Electroconvulsive therapy for self-injurious behaviour in autism spectrum disorders: recognizing catatonia is key. Curr Opin Psychiatry 2018, 31:116-122, doi: 10.1097/YCO.0000000000000393

21. Borisovskaya A, Bryson WC, Buchholz J, Samii A, Borson S. Electroconvulsive therapy for depression in Parkinson's disease: systematic review of evidence and recommendations.
Neurodegener Dis Manag 2016, 6:161-176, doi: 10.2217/nmt2016-0002

22. Popeo D, Kellner CH. ECT for Parkinson's disease. Med Hypotheses 2009, 73:468-469, doi: 10.1016/j.mehy.2009.06.053

23. Pinchotti DM, Abbott C, Quinn DK. Targeted Electroconvulsive Therapy for Super Refractory Status Epilepticus: A Case Report and Literature Review. Psychosomatics 2018, 59:302-305, doi: 10.1016/j.psym.2017.10.004

24. Zeiler FA, Matuszczak M, Teitelbaum J, Gillman LM, Kazina CJ. Electroconvulsive therapy for refractory status epilepticus: A systematic review. Seizure 2016, 35:23-32, doi: 10.1016/j. seizure.2015.12.015

25. Van den Berg JF, Kruithof HC, Kok RM, Verwijk E, Spaans HP. Electroconvulsive Therapy for Agitation and Aggression in Dementia: A Systematic Review. Am J Geriatr Psychiatry 2018, 26:419-434, doi: 10.1016/j.jagp.2017.09.023

26. Allen RM. Pseudodementia and ECT. Biol Psychiatry 1982, 17:1435-1443, PMID: 7159640

27. Rapinesi C, Serata D, Del Casale A, Kotzalidis GD, Mazzarini L, Fensore C. Depressive pseudodementia in the elderly: effectiveness of electroconvulsive therapy. Int J Geriatr Psychiatry. 2013, 28:435-438, doi: $10.1002 /$ gps.3877

28. Kellner $\mathrm{CH}$, Greenberg RM, Murrough JW, Bryson EO, Briggs MC, Pasculli RM. ECT in treatment-resistant depression. Am $J$ Psychiatry 2012, 169(12):1238-1244, doi: 10.1176/appi.ajp. 2012.12050648

29. Papakostas Y, Stefanis C, Sinouri A, Trikkas G, Papadimitriou G, Pittoulis $\mathrm{S}$. Increases in prolactin levels following bilateral and unilateral ECT. Am J Psychiatry 1984, 141:1623-1624, PMID: 6507669

30. Papakostas Y, Stefanis C, Markianos M, Papadimitriou G. Electrode Placement and Prolactin Response to Electroconvulsive Therapy. Convuls Ther 1986, 2:99-107, PMID: 11940852

31. Papakostas Y, Markianos M, Papadimitriou G, Stefanis C. Prolactin response induced by ECT and TRH. Br J Psychiatry 1986, 148:721-723, PMID: 3096413

32. Papakostas $Y$, Markianos M, Papadimitriou G, Stefanis C. Thyrotropin and Prolactin Secretion during ECT: Implications for the Mechanism of ECT Action. Convuls Ther 1990, 6:214-220, PMID: 11941070

33. Zervas IM, Papakostas YG, Theodoropoulou MA, Dimitrakopoulos $\mathrm{C}$, Vaidakis N, Daras $\mathrm{M}$. Thyrotropin-releasing hormone administration does not affect seizure threshold during electroconvulsive therapy. J ECT 2003, 19:136-138, PMID: 12972982

34. Papakostas Y, Markianos M, Pehlivanidis A, Papadimitriou GN, Zervas IM, Daras $\mathrm{M}$ et al. Effects of thyrotropin-releasing hormone administration on the electroconvulsive therapy induced prolactin responses and seizure time. Biol Psychiatry 1996, 15, 39:444-447, PMID: 8679790

35. Zervas IM, Pehlivanidis AA, Papakostas YG, Markianos M, Papadimitriou GN, Stefanis CN. Effects of TRH administration on orientation time and recall after ECT. J ECT 1998 14(4):236240, PMID: 9871843 
36. Papakostas YG, Markianos M, Pehlivanidis A, Zervas IM, Papadimitriou GN, Stefanis C. Blunted TSH response to TRH and seizure duration in ECT. Acta Psychiatr Scand 1999, 99:6872, PMID: 10066009

37. Fink M, Nemeroff CB A Neuroendocrine View of ECT. Convuls Ther 1989, 5:296-304, PMID: 11941027

38. Swartz CM. Electroconvulsive and Neuromodulation Therapies. Cambrigde: Cambridge University Press, 2009

39. Esel E, Kose K, Hacimusalar Y, Ozsoy S, Kula M, Candan Z, Turan $T$. The effects of electroconvulsive therapy on GABAergic function in major depressive patients. J ECT 2008, 24:224-228, doi: 10.1097/YCT.0b013e31815cbaa1

40. Zeiler FA, Matuszczak M, Teitelbaum J, Gillman LM, Kazina CJ. Electroconvulsive therapy for refractory status epilepticus: A systematic review. Seizure 2016, 35:23-32, doi: 10.1016/j. seizure.2015.12.015

41. Pinchotti DM, Abbott C, Quinn DK. Targeted Electroconvulsive Therapy for Super Refractory Status Epilepticus: A Case Report and Literature Review. Psychosomatics 2018, 59:302-305, doi: 10.1016/j.psym.2017.10.004

42. Polyakova M, Stuke K, Schuemberg K, Mueller K, Schoenknecht $\mathrm{P}$, Schroeter ML. BDNF as a biomarker for successful treatment of mood disorders: a systematic \& quantitative meta-analysis. $J$ Affect Disord 2015, 174: 432-440, doi: 10.1016/j.jad.2014.11.044

43. Polyakova M, Schroeter ML, Elzinga BM, Holiga S, Schoenknecht $P$, de Kloet ER. Brain-Derived Neurotrophic Factor and Antidepressive Effect of Electroconvulsive Therapy: Systematic Review and Meta-Analyses of the Preclinical and Clinical Literature. PLoS One 2015, 3, 10:e0141564, doi: 10.1371/journal.pone.0141564, eCollection 2015

44. Nibuya M, Morinobu S, Duman RS. Regulation of BDNF and trkB mRNA in rat brain by chronic electroconvulsive seizure and antidepressant drug treatments. J Neurosci 1995, 15:75397547, PMID: 7472505

45. Newton SS, Collier EF, Hunsberger J, Adams D, Terwilliger R, Selvanayagam E, Duman RS. Gene profile of electroconvulsive seizures: induction of neurotrophic and angiogenic factors. J Neurosci 2003, 23:10841-10851, PMID: 14645477

46. Altar CA, Laeng P, Jurata LW, Brockman JA, Lemire A, Bullard $\mathrm{J}$, et al Electroconvulsive seizures regulate gene expression of distinct neurotrophic signaling pathways. J Neurosci 2004, 24:2667-2677

47. Dyrvig M, Christiansen SH, Woldbye DP, Lichota J. Temporal gene expression profile after acute electroconvulsive stimulation in the rat. Gene 2014, 539:8-14, doi: 10.1016/j.gene.2014.01.072

48. Kaneko T, Kanazawa T, Nishiguchi M, Kikuyama H, Tsutsumi A, Uenishi H. Microarray Analysis of Human Blood during Electroconvulsive Therapy. J ECT 2015, 31:234-237, doi: 10.1097/ YCT. 0000000000000234

49. de Jong JO, Arts B, Boks MP, Sienaert P, van den Hove DL, Kenis $\mathrm{G}$ et al. Epigenetic effects of electroconvulsive seizures. J ECT 2014, 30:152-159, doi: 10.1097/YCT.0000000000000141

50. Dyrvig M, Gøtzsche CR, Woldbye DP, Lichota J. Epigenetic regulation of Dnmt3a and Arc gene expression after elec- troconvulsive stimulation in the rat. Mol Cell Neurosci 2015, 67:137-143, doi: 10.1016/j.mcn.2015.06.011

51. Bolwig TG. Neuroimaging and electroconvulsive therapy: a review. J ECT 2014, 30:138-142, doi: 10.1097/YCT.0000000000000140

52. Argyelan M, Lencz $T$ Kaliora S, Sarpal DK, Weissman N, Kingsley PB et al. Subgenual cingulate cortical activity predicts the efficacy of electroconvulsive therapy. Trans/ Psychiatry 2016, 6:e789, doi: 10.1038/tp.2016.54

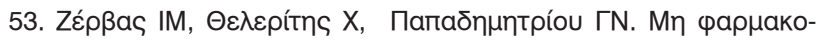

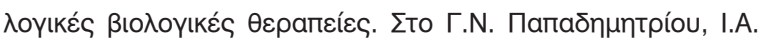

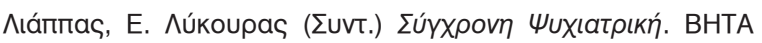

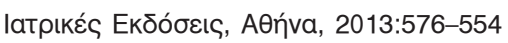

54. Taylor WD. Clinical practice depression in the elderly. N Engl J Med 2014, 371:1228-1236, doi: 10.1056/NEJMcp1402180

55. Unützer J, Park M. Older adults with severe, treatment-resistant depression. JAMA 2012, 308:909-918, doi: 10.1001/2012. jama. 10690

56. Kellner $\mathrm{CH}$, Husain M, Knapp RG, McCall WV, Petrides G, Rudorfer MV. Right Unilateral Ultrabrief Pulse ECT in Geriatric Depression: Phase 1 of the PRIDE Study. Am J Psychiatry 2016, 173:1101-1109

57. Leiknes KA, Cooke MJ, Jarosch-von Schweder L, Harboe I, Høie B. Electroconvulsive therapy during pregnancy: a systematic review of case studies. Arch Womens Ment Health 2015, 18:1-39, doi: 10.1007/s00737-013-0389-0

58. Ward HB, Fromson JA, Cooper JJ, De Oliveira G, Almeida M. Recommendations for the use of ECT in pregnancy: literature review and proposed clinical protocol. Arch Womens Ment Health 2018, 21:715-722, doi: 10.1007/s00737-018-0851-0

59. Rey J.M, Walter G. Half a century of ECT use in young people. Am J Psychiatry 1997, 154:595-602, PMID: 9137112

60. Bloch Y, Levcovitch Y, Bloch AM, Mendlovic S, Ratzoni G. Electroconvulsive therapy in adolescents: similarities to and differences from adults. J Am Acad Child Adolesc Psychiatry 2001, 40:1332-1336, doi: 10.1097/00004583-200111000-00014

61. Ghaziuddin N, Kutcher SP, Knapp P, Bernet W, Arnold V, Beitchman J. Practice parameter for use of electroconvulsive therapy with adolescents. J Am Acad Child Adolesc Psychiatry 2004, 43:1521-1539

62. Bush G, Fink M, Petrides G, Dowling F, Francis A. Catatonia. II. Treatment with lorazepam and electroconvulsive therapy. Acta Psychiatr Scand 1996, 93:137-143, PMID: 8686484

63. Consoli A, Benmiloud M, Wachtel L, Dhossche D, Cohen D, Bonnot O. Electroconvulsive therapy in adolescents with the catatonia syndrome: efficacy and ethics. J ECT 2010, 26:259265, doi: 10.1097/YCT.0b013e3181fb3924

64. Flamarique I, Castro-Fornieles J, Garrido JM, de la SE, Pons $A$, Bernardo $M$ et al. Electroconvulsive therapy and clozapine in adolescents with schizophrenia spectrum disorders: is it a safe and effective combination? J Clin Psychopharmacol 2012, 32:756-766, doi: 10.1097/JCP.0b013e318270e2c7

65. Chanpattana W, Kramer BA, Kunigiri G, Gangadhar BN, Kitphati $\mathrm{R}$, Andrade C. A survey of the practice of electroconvulsive 
therapy in Asia. J ECT 2010, 26:5-10, doi: 10.1097/YCT. ob013e3181a74368

66. Schweder LJ, Lydersen S, Wahlund B, Bergsholm P, Linaker OM. Electroconvulsive therapy in Norway: rates of use, clinical characteristics, diagnoses, and attitude. J ECT 2011, 27: 292-295, doi: 10.1097/YCT.0b013e318208e24b

67. Teh SP, Xiao AJ, Helmes E, Drake DG. Electroconvulsive therapy practice in Western Australia. J ECT 2005, 21:145-150, PMID: 16127302

68. Thompson JW, Blaine JD. Use of ECT in the United States in 1975 and 1980. Am J Psychiatry 1987, 144:557-562, PMID: 3578563

69. Cohen D, Taieb O, Flament M, Benoit N, Chevret S, Corcos M. et al. Absence of cognitive impairment at long-term follow-up in adolescents treated with ECT for severe mood disorder. Am J Psychiatry 2000, 157:460-462, doi: 10.1176/appi.ajp.157.3.460

70. Ghaziuddin N, Laughrin D, Giordani B. Cognitive side effects of electroconvulsive therapy in adolescents. $J$ Child Adolesc Psychopharmacol 2000, 10:269-276, doi: 10.1089/cap. 2000.10.269

71. Husain MM, Rush AJ, Fink M, Knapp R, Petrides G, Rummans. Speed of response and remission in major depressive disorder with acute electroconvulsive therapy (ECT): a Consortium for Research in ECT (CORE) report. J Clin Psychiatry 2004, 65:485-491, PMID: 15119910

72. Kolshus E, Jelovac A, McLoughlin DM. Bitemporal v. highdose right unilateral electroconvulsive therapy for depression: a systematic review and meta-analysis of randomized controlled trials - CORRIGENDUM. Psychol Med 2018, 48:703-704, doi: $10.1017 /$ S0033291717002720

73. Kellner CH, Farber KG. The Role of Bilateral ECT When Right Unilateral ECT Is Inferior. Am J Psychiatry 2016, 173:731, doi: 10.1176/appi.ajp.2016.16020252.

74. Lapidus $\mathrm{KA}$, Kellner $\mathrm{CH}$. When to switch from unilateral to bilateral electroconvulsive therapy. J ECT 2011, 27:244-246, doi: $10.1097 /$ YCT.0b013e31820059e1

75. Kellner CH, Farber KG, Chen XR, Mehrotra A, Zipursky GDN. A systematic review of left unilateral electroconvulsive therapy. Acta Psychiatr Scand 2017, 136:166-176, doi: 10.1111/acps. 12740

76. Ren L, Deng J, Min S, Peng L, Chen Q. Ketamine in electroconvulsive therapy for depressive disorder: A systematic review and meta-analysis. J Psychiatr Res 2018, 104:144-156, doi: 10.1016/j.jpsychires.2018.07.003

77. Mirzakhani H, Welch CA, Eikermann M, Nozari A. Neuromuscular blocking agents for electroconvulsive therapy: a systematic review. Acta Anaesthesiol Scand 2012, 56:3-16, doi: 10.1111/j.1399-6576.2011.02520.x

78. Wang W, Pu C, Jiang J, Cao X, Wang J, Zhao M et al. Efficacy and safety of treating patients with refractory schizophrenia with antipsychotic medication and adjunctive electroconvulsive therapy: a systematic review and meta-analysis. Shanghai Arch Psychiatry 2015, 27:206-219, doi: 10.11919/j.issn.10020829.215093
79. Pippard J. Audit of electroconvulsive treatment in two National Health Service regions. Br J Psychiatry 1992, 160:621-637, PMID: 1591572

80. Abrams R. Electroconvulsive Therapy. Oxford University Press, New York, 2002

81. Petrides G, Fink M The "half-age" stimulation strategy for ECT dosing. Convuls Ther 1996, 12:138-146

82. Sackeim HA, Devanand DP, Prudic J. Stimulus intensity, seizure threshold, and seizure duration: impact on the efficacy and safety of electroconvulsive therapy. Psychiatr Clin North Am 1991, 14:803-843, PMID: 1771150

83. Tørring N, Sanghani SN, Petrides G, Kellner $\mathrm{CH}$, Østergaard $\mathrm{SD}$. The mortality rate of electroconvulsive therapy: a systematic review and pooled analysis. Acta Psychiatr Scand 2017, 135:388-397, doi: 10.1111/acps. 12721

84. Andrade C, Arumugham SS, Thirthalli J. Adverse Effects of Electroconvulsive Therapy. Psychiatr Clin North Am 2016, 39:513-530, doi: 10.1016/j.psc.2016.04.004

85. Tzabazis A, Schmitt HJ, Ihmsen H, Schmidtlein M, Zimmermann R, Wielopolski J, Mónster T. Postictal agitation after electroconvulsive therapy: incidence, severity, and propofol as a treatment option. J ECT 2013, 29:189-195, doi: 10.1097/YCT. 0b013e3182887b5b

86. Sobin C, Sackeim HA, Prudic J, Devanand DP, Moody BJ, McElhiney MC. Predictors of retrograde amnesia following ECT. Am J Psychiatry 1995, 152:995-1001, doi:10.1176/ajp.152.7.995

87. Steif BL, Sackeim HA, Portnoy S, Decina P, Malitz S. Effects of depression and ECT on anterograde memory. Biol Psychiatry 1986, 21:921-930

88. Sackeim HA, Haskett RF, Mulsant BH, Thase ME, Mann JJ, Pettinati et al. Continuation pharmacotherapy in the prevention of relapse following electroconvulsive therapy: a randomized controlled trial. JAMA 2001, 285:1299-1307, PMID: 11255384

89. Kellner CH, Knapp RG, Petrides G, Rummans TA, Husain MM, Rasmussen K. Continuation electroconvulsive therapy vs pharmacotherapy for relapse prevention in major depression: a multisite study from the Consortium for Research in Electroconvulsive Therapy (CORE). Arch Gen Psychiatry 2006, 63:1337-1344, doi: 10.1001/archpsyc.63.12.1337

90. Kellner CH, Husain MM, Knapp RG, McCall WV, Petrides G, Rudorfer MV. A Novel Strategy for Continuation ECT in Geriatric Depression: Phase 2 of the PRIDE Study. Am J Psychiatry. 2016, 173:1110-1118, doi:10.1176/appi.ajp.2016.16010118

91. Prudic J, Haskett RF, McCall WV, Isenberg K, Cooper T, Rosenquist PB. Pharmacological strategies in the prevention of relapse after electroconvulsive therapy. J ECT 2013, 29:3-12, doi: 10.1097/YCT.0b013e31826ea8c4

92. Papadimitriou GN, Zervas IM, Papakostas YG. Unilateral ECT for prophylaxis in affective illness. J ECT 2001, 17:229-231, PMID: 11528326

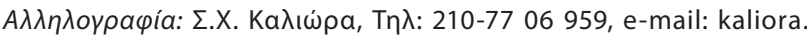
iatreio@gmail.com 